\title{
Zur Kenntnis der extrafloralen Nektarien.
}

\section{INAUGURAL-DISSERTATION ZUR}

ERLANGUNG DER DOKTORWÜRDE

DER

HOHEN PHILOSOPHISCHEN FAKULTÄT

DER

GEORG-AUGUST-UNIVERSIT ÄT

ZU

GÖTTINGEN

VORGELEGT VON

EDUARD SCHWENDT

AUS

SEGGERDE.

Mit 2 Tafeln.

GÖTTINGEN

1906. 
Tag der mündlichen Prüfung: 25. Juli 1906.

Referent: Herr Professor Dr. A. Peter.

Sonderabdruck aus „Beihefte zum Botan. Centralblatt“ Bd. XXII (1907) Abt. I. Verlag C. Heinrich, Dresden-N. 


\section{Meinen lieben Eltern}

in Dankbarkeit

gewidmet.

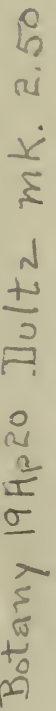


Digitized by the Internet Archive in 2017 with funding from

University of Illinois Urbana-Champaign Alternates 


\section{Inhalt.}

I. Vorbemerkungen

Historische Literaturangaben . . . . . . . . . . . 7

Vorbemerkungen zur Nomenklatur und Präparation . . . . 9

Verzeichnis der bearbeiteten Pflanzenarten. . . . . . . . 10

II. Anatomisch - entwicklungsgeschichtliche Untersuchungen

Muehlenbeckia sagittifolia. . . . . . . . . . . . . . . 11

Polygonum baldschuanicum . . . . . . . . . . . . . . 14

Einige andere Polygonaceen . . . . . . . . 15

Gossypium brasiliense . . . . . . . . . . . . . . . . . 16

Einige weitere Gossypien . . . . . . . . . . . 18

Tecoma radicans. . . . . . . . . . . . . . . . . . . 18

Ligustrum Regelianum, Syringa sp. . . . . . . . . . . . . 21

Polypodiaceen

a) Drynaria Linnaei . . . . . . . . . . . . . 24

b) Drynaria quercifolia . . . . . . . . . . . . . 26

c) Weitere Polypodiaceen . . . . . . . . . . . . 26

Acacia cornigera . . . . . . . . . . . . . . . . . . . 26

III. Zusammenfassung und Kritik

1. Verbreitung der Nektarien; Drüsengewebe, sezernierende Epidermis, Gefäßbündel . . . . . . . . . . . 31

2. Lageverhältnis, meristemat. Charakter des Nektariumgewebes ' 33

3. Verdickter und verkorkter Stielteil der nektarabsondernden Trichome . . . . . . . . . . . . 34

4. „Verholzte" Scheide. . . . . . . . . . . . . 35

5. Inhalt, Gerbstoff . . . . . . . . . . 36

6. Vorkommen homologer Gebilde . . . . . . . . . . . 37

7. Bedeutung der extrafloralen Nektarien . . . . . . 39

IV. Einige Ergebnisse . . . . . . . . . . . . . 42

V. Literaturverzeichnis . . . . . . . . . . 43

VI. Figurenerklärung . . . . . . . . . . . . . . . 45 



\section{Zur Kenntnis der extrafloralen Nektarien.}

Von

\section{Eduard Schwendt.}

Mit 2 Tafeln.

\section{Vorbemerkungen.}

Von den biologischen Problemen, die augenblicklich ihrer Lösung harren, haben wenige so sehr das allgemeine Interesse erregt, als die Wechselbeziehungen zwischen Pflanzen und Ameisen. Mit diesen Beziehungen haben viele Autoren die Deutung der extrafloralen Nektarien in Verbindung gebracht, und hierin liegt wohl vor allem der Grund, daß die Literatur über diese Organe bereits einen ansehnlichen Umfang erreicht hat. Doch nicht nur von dieser rein biologischen Seite, sondern auch von manchen anderen Gesichtspunkten aus sind die extrafloralen Nektarien in den letzten Jahrzehnten oft der Gegenstand wissenschaftlicher Untersuchung gewesen. Haben nun auch manche der dabei aufgekommenen Fragen bereits eine Aufklärung erfahren, so ist unsere Kenntnis der Nektarien in anatomischer oder gar entwicklungsgeschichtlicher Hinsicht doch noch sehr lückenhaft. Daher stellte ich mir in der vorliegenden Arbeit dic Aufgabe, vor allem nach diesen Richtungen hin einen Beitrag zu liefern, der bei einer späteren zusammenfassenden Darstellung dieses Gegenstandes vielleicht von einigem Nutzen sein dürfte.

Die Untersuchungen, über welche nachstehend berichtet wird, sind von mir im Göttinger botanischen Institut auf Veranlassung des Herrn Professor Dr. A. Peter ausgeführt worden. Es ist mir eine angenchme Pflicht, diesem meinem verehrten Lehrer meinen herzlichen Dank auszusprechen für das mir stets entgegengebrachte Interesse und die wertvolle Unterstützung, die er mir während dieser Arbeit zuteil werden ließ.

\section{Kurzer Überblick über die historische Entwicklung unserer Kenntnisse von den extrafloralen Nektarien.}

Das Vorkommen extrafloraler Nektarien ist lïngst bekannt: es sind die mannigfachen "glandulae" der alten Systematiker, die 
bei neueren Forschungen zum großen Teil sich als Nektarien erwiesen.

Zuerst machte Chr. K. Sprengel $\left.{ }^{1}\right) 1793$ auf das Vorkommen und die drüsige Eigenschaft einiger extrafloraler "Saftdrüsen" aufmerksam.

Die erste größere anatomische Bearbeitung der extrafloralen Nektarien rührt von Caspary ${ }^{2}$ her, der in seiner 1848 erschienenen Dissertation "De nectariis" ein besonderes Kapitel den "nectariis extra florem sitis" widmete und damit zugleich der Urheber dieses Terminus wurde.

Von den seitdem erschienenen Arbeiten - eine größere Anzahl findet sich in dem beigegebenen Literaturverzeichnis angeführt - , die anatomische Verhältnisse berücksichtigen, erwähne ich nur die wichtigsten und beschränke mich auf ganz kurze Angaben, da eine eingehende Besprechung weit über den Rahmen der vorliegenden Arbeit hinausgreifen würde.

Im Jahre 1876 veröffentlichte Reinke ${ }^{3}$ ) die Ergebnisse einer Untersuchung über die anatomische Struktur der an Laubblättern befindlichen Sekretionsorgane, in der er auch die Anatomie und Entwicklungsgeschichte einiger Nektarien behandelt hatte.

V. A. Poulsen ${ }^{4}$ ) gab in den folgenden Jahren eine Reihe kleinere, zumteil mit Abbildungen versehene Mitteilungen, die leider öfters nicht eingehend genug sind, im übrigen auch manchen deutschen Forschern unbekannt blieben.

Wesentlich gefördert wurden unsere Kenntnisse durch B o n n i e $\mathrm{r}^{5}$ ), der sein Hauptangenmerk der physiologischen Seite zuwandte, doch auch in anatomischer Hinsicht sich Verdienste erwarb. Seit dem Erscheinen von D elp in $0 \mathrm{~s}^{6}$ ) "Funzione mirmecofila nel regno vegetale“ beschäftigten sich verschiedene italienische Forscher mit Untersuchungen über ,nettarii estranuziali“; ich will hier unter anderen nur Morini7) nennen, der in seiner Abhandlung die bis dahin gewonnenen Kenntnisse zusammenfaßte und durch eigene Untersuchungen vervollständigte.

Außer den kleineren Arbeiten von Rathay ${ }^{8}$ ) und v. Wett-

1) Ch. K. Sprengel, Das entdeckte Geheimnis der Natur im Bau und in der Befruchtung der Blumen. Berlin 1793.

2) Caspary, De nectariis. Dissert. Elverfeldae 1848.

3) Reinke, Beiträge zur Anatomie der an Laubblättern, besonders an den Zähnen derselben vorkommenden Sekretionsorgane. Pringsheims Jahrb. für wiss. Bot. Bd. 10. $187 t$.

4) V. A. Poulsen, Om nogle Trikomer og Nektarier. Videnskabelige Meddelelser fra den naturh. Forening Kjöbenhavn 1875.

5) Bonnier, Les nectaires. Ann. des sciences naturelles 1878. Serie 6, Tome 8. Paris 1878.

${ }_{6}^{6}$ Delpino, Funzione mirmecofila nel regno vegetale. Memorie della accademia di Bologna. Serie IV. $1886-88$.

7) Morini, Contributo all' anatomia ed alla fisiologia dei nettarii estranuziali. Memorie della accademia di Bologna. Serie IV, Tomo 7. Bologna 1886.

$\left.{ }^{8}\right)$ Rathay, Utber nektarabsondernde Trichome einiger Melampyrumarten. Sitzber. d. k. Ak. d. Wiss. Wien, Math. naturw. Classe. Bd. 81. Wien 1880. 
stein $^{1}$ ) ist hier noch die von Correns' ${ }^{2}$ zu erwähnen, der die Anatomie und Entwicklungsgeschichte der extrafloralen Nektarien von Dioscorea eingehend untersuchte.

Mit dem Erscheinen der Dissertation von Aufrecht ${ }^{3}$ ) schließen die anatomischen Untersuchungen meines Wissens ab.

\section{Bemerkungen über das untersuchte Material, die Präparation und die Terminologie.}

In Anbetracht der außerordentlich großen Zahl der extrafloralen Nektarien ist bis jetzt nur ein recht geringer Bruchteil derselben anatomisch näher bekannt geworden. Zum großen Teil sind es leicht zugängliche und auffallende Drïsen, die in vielen Untersuchungen inmer wiederkehren; ich will hier nur auf Pteris aquilina hinweisen, dessen Nektarien, soweit mir bekannt wurde, nicht weniger als fünfmal bearbeitet worden sind; mit Ricimus, Sambucus, Viburnum u. a. ist es ähnlich. Ich behandle daher im folgenden aus einer größeren Untersuchungsreihe (etwa 60 Arten) nur solche Spezies genauer, deren feinere Struktur noch gar nicht oder doch nur oberflächlich und von anderen Gesichtspunkten aus untersucht ist. Besondere Beachtung wendete ich solchen Drüsen zu, die mittels Trichombildungen sezernieren.

Das von mir untersuchte Material wurde fast durchweg in Paraffin eingebettet, je nach Bedürfnis in Schnitte von 4 bis $15 \mu$ Dicke zerlegt und meist mit Delafield'schem Hämatoxylin gefärbt. Die Untersuchung, die zwei Jahre in Anspruch nahm, insbesondere diejenige der Entwicklungsgeschichte, wurde an Schnittserien durchgeführt. Meistens wurden von einem Objekt mehrere Serien angefertigt, sodaß die Gesantzahl der studierten Schnitte sich auf etwa 20000 beläuft.

Bevor ich mich der Darstellung der Einzeluntersuchungen zuwende, wird es angesichts der etwas unsicheren Terminologie zweckmäßig sein, einige Bemerkungen über dieselbe hier vorauszuschicken.

In einem großen Teil der Arbeiten der letzten Jahre ist von extranuptialen Nektarien die Rede: eine von Delpino eingeführte rein biologische Bezeichnung, die ich nicht acceptieren möchte, weil die in ihr liegende einschränkende Zweckbestimmung meines Erachtens erst von Fall zu Fall zu beweisen ist. Ich behalte daher den alten einmal eingebürgerten Ausdruck extrafloral bei, obwohl auch dieser wenig positives aussagt, und auch namentlich inbezug auf die Nektarien der Farne durchaus nicht einwandfrei ist.

Was den von Linné eingefïhrten Terminus Nektarium anbelangt - in der vorliegenden Arbeit öfter kurz I)rïse genannt -

1) v. Wettstein, Über die Compositen der österreichisch-ungarischen Flora mit zuckerabscheidenden Hüllschuppen. Sitzber. Wiener Akad. Bd. 97. 1888.

${ }^{2}$ ) Correns, Zur Anatomie u. Entwicklungsgeschichte der extranuptialen Nektarien von Ilioscorea. Sitzber. d. Akad. zu Wien. Math.-naturw. Classe. Bd. 97. Wien 1888.

3) A ufrecht, Beitrag z. Kenntnis extrafloraler Nektarien. Diss. Zür. 1891. 
so ist es nicht möglich, für diesen Kollektivbegriff eine strenge morphologisch-anatomische Definition zu geben, da er gerade in Bezug auf die extrafloralen Fälle ein Sammelname für die heterogensten Gebilde ist, denen allen nur die physiologische Funktion der Nektarabsonderung gemeinsam ist. Ich möchte die Anwendung dieses Ausdruckes einschränken auf solche drüsig ausgebildete, auf vegetativen Teilen der Pflanze befindliche Stellen, an denen normalerweise die Absonderung einer zuckerhaltigen Flüssigkeit (Nektar) erfolgt.

Von einem Nektariumgewebe oder Drüsengewebe rede ich im folgenden nur, wenn ein solches sowohl inhaltlich als auch an tomisch scharf ausgeprägt ist.

\section{Bearbeitete Arten.}

Geschnitten wurden die extrafloralen Nektarien bezw. nektarienähnlichen Drüsen von folgenden Arten:

1) Farne: Drynaria Linnaei Bory, D. quercifolia Bory, D. rigidula J. Sm., Polypodium Heracleum Kze., P. Meyenianum Schott, Pteris aquilina Linn.;

2) Dioscoreaceen: Dioscorea alata Linn.;

3) Salicaceen: Populus alba Linn., P. candicans Ait. Hort. Kew, P. monilifera Ait. Hort. Kew, P. tremula Linn.;

4) Polygonaceen: Muehlenbeckia sagittifolia Meissn., Polygonum baldschuanicum Regel, P. Convolvuhs Linn., P. dumetorum Linn., P. multiflormu Thunb.;

5) Euphorbiaceen: Hippomane Mancinella Linn., Hura crepitans Linn., Ricinus commumis Linn.;

6) Turneraceen: Tuinera angustifolia Mill.;

7) Rosaceen: Prums Armeniaca Linn., P. avium Linn., P. Laurocerasus Linn., P. Padus Linn.;

8) Passifloraceen: Modecca lobata Jacq., Passiflora coemulea Linn., P. edulis Sims.;

9) Maliaceen: Gossypim brasiliense Macfad., G. Davidsonii Kellogg, G. herbaceum Linn., G. microcarpum Tod.;

10) Cucurbitaceen: Actinostemma paniculatum Maxim., Luffa cylindrica M. Roem., Momordica cochinchinensis Spreng.;

11) Leguminosen: Acacia comigera Willd., A. Lichtensteinii hort., A. longifolia Willd., A. longifolia var. Sophorae, A. lophantha Willd., A. neriifolia A. Cunn., A. saligna Wendl., Albizsia Nenmamiana Heynt, Cassia arborescens Mill., C. marylandica Linn., Vicia amphicarpa Dorthes, V. Faba Linn., V. sepium Linn.;

12) Bignoniaceen: Tecoma radicans Juss.;

13) Verbenaceen: Clerodendion fragrans Willd., Cl. ixoriflorum Hassk.;

14) Oleaceen: Ligustrum lucidum Mill., L. Regeliamum Hort. Lemoine, L. vulgare Linn., Syciuga chinensis Willd., S. persica Linn.;

15) Plumbagineen: Plumbago rosea Linn.;

16) Caprifoliaceen: Sambucus uigra Linn.; S. racemosa Linn., Viburnum Opulus Linn. 
Interessante Verhältnisse zeigen unter anderen die Nektarien von Actinostemma pariculatum und Plumbago rosea, doch sind die Untersuchungen noch nicht so weit gediehen, daß sie hier veröffentlicht werden konnten.

\section{Anatomisch-entwicklungsgeschichtliche Untersuchungen.}

\section{Muehlenbeckia sagittifolia Meissn. - Sïdanerika.}

a) Ausgebildetes Nektarium.

An der Unterseite des Blattkissens befindet sich das extraflorale Nektarium als eine flache, bis $1 \mathrm{~mm}$ breite und $2 \mathrm{~mm}$ lange, meist elliptisch begrenzte Grube, die von den angrenzenden hypodermalen Gewebe wallartig rings umgeben wird. An warmen Tagen wurde an dieser Stelle reichlich eine Flüssigkeit ausgeschieden, die Fehlings Lösung zu Kupferoxydul reduzierte.

Für die nachfolgende Untersuchung ist, sofern nichts anderes bemerkt wird, ein sezernierendes Nektarium zugrunde gelegt.

Soweit die grubenartige Einsenkung reicht, sind die hypodermalen Zellen des Blattpolsters, sowie noch etwa fünf weitere darunter liegende Zellschichten kleiner, mehr polyedrisch gestaltet und dichter aneinanderstoßend, als es sonst an entsprechenden Stellen des Blattkissens der Fall ist; inhaltlich jedoch weichen sie von ihrer Umgebung nicht in dem Maße ab, daß man hier von einem typischen Drüsengewebe reden könnte, in welchen man die Entstehung des Nektars zu suchen hätte. Bisweilen scheinen sich die Phloemelemente der Gefäßbündel des Blattkissens diesem eben genannten Gewebe etwas zu nähern. Der Grund der Vertiefung ist mit zahlreichen Trichomen bedeckt, deren Gesamtheit - etwa 30 bis 70 - das Nektarium bilden, das demnach in diesem Falle ein rein epidermales Gebilde, eine Hautdrüse im Sinne de Bary's ist.

An dem einzelnen Trichom oder Drüsenhaar muß man auf Grund der Entwicklungsgeschichte dreierlei unterscheiden: 1) den Fuß- oder Basalteil, 2) den Stiel, 3) das Köpfchen oder den sezernierenden 'Teil.

Die Basalzellen (Fig. 1b) bilden die Fortsetzung der Epidermis des Blattkissens. Der Fub des einzelnen Drüsenhaares ist als solcher im ausgebildeten Zustande schwer zu erkennen; er besteht bei mittelgroßen Trichomen aus etwa 50 bis 70 \%ellen.

Der nach außen daranschließende Stielteil (Fig. 1, s) ist meist einschichtig und gleichfalls aus etwa 60 kubischen Zellen zusammengesetzt. Während die Basalzellen der einzelnen I)rüsenhaare in innigem Zusammenhang stehen, lassen die Stielteile zwischen den einzelnen Trichomen kleine Zwischenräume (Fig. 1, l) frei. Diese Stielzellen sind plasmareich und treten recht deutlich auf mit Hämatoxylin gefärbten Schnitten 
dadurch hervor, daß ihre Radialwände nicht tingiert werden. Letztere sind etwas verdickt und werden mit Chlorzinkjod gelbbraun, sind also ,verkorkt"; zeitweise bemerkt man auch an den Tangentialwänden, die nach dem FuB hin liegen, eine schwache „Verkorkung“. Behandelt man Schnitte nit Fuchsinlösung, so werden die Radialwände intensiv rot gefärbt, die das 'Trichom kontinuierlich bekleidende Cuticula aber ist als zartes farblos bleibendes Häutchen bei starker Vergrößerung deutlich außerhalb der Radialwände des Stiels zu erkennen. Aus welchen Stoffen die eben als "verkorkt" bezeichnete Membran besteht, ist nach dem heutigen Stande unserer mikrochemischen Kenntnisse nicht zu entscheiden, jedenfalls erhellt aus dem soeben angegebenen Befund, daß sie chemisch oder doch mindestens physikalisch mit der Cuticula nicht identisch ist.

Bei hinreichend dünnen Flächenschnitten kann man häufig an der Stärke der radialen Wandverdickungen noch die Reihenfolge ihrer successiven Entstehung erkennen (Fig. 2), besonders die durch die ersten Radialteilungen (Quadrantenbildung) gebildeten Membranen sind fast in jedem Drüsenhaar auch auf Querschnitten durch ihre Dicke vor allen anderen Zellwänden ausgezeichnet.

Der Köpfchenteil oder sezernierende Abschnitt des Haargebildes besteht in der Regel aus einer Schicht langgestreckter zartwandiger Palissadenzellen, die reich an Plasma sind und relativ große Kerne besitzen. Ihre Zahl ist ziemlich groß, etwa 150 bis 180, sodaß das einzelne Trichom dennach im ganzen etwa 320 Zellen enthält. Bekleidet wird das Köpfchen von einer zarten Cuticula.

Ein auf dem Stadium der Sekretion mit Kaliumbichromat ${ }^{1}$ ) behandeltes Nektarium läßt einen enormen Reichtum an "Gerbstoff." erkennen: Palissaden-, Stiel- und Basalteil der einzelnen Trichome enthalten ungewöhnlich viel Gerbstoff, der in den Stielzellen in Form kleiner Kugeln, in den beiden anderen Teilen des Drüsenhaares in größeren Ballen ausgefällt ist. Auch die subepidermale Schicht ist sehr gerbstoffreich. Darunter folgen, soweit sich die grubige Einsenkung erstreckt, ein bis zwei gerbstofffreie Schichten; in tieferen Zelllagen ist er wieder vorhanden, wie denn überhaupt in der ganzen Pflanze die Körper aus der Klasse der Gerbstoffe weit verbreitet sind. Der Gerbstoff ist von den ersten Entwicklungszuständen an im Nektarium vorhanden, wandert also nicht etwa erst später ein; mit dem Wachstum der Drüse ninımt auch die Menge des Gerbstoffes zu.

\section{b) Entwicklungsgeschichte.}

Wie die nachfolgende Darstellung der Entwicklungsgeschichte lehrt, sind die einzelnen Nektar ausscheidenden Febilde

1) Zum Gerbstoffnachweis wurden die Objekte meist nach der von Berthold: Untersuchungen zur Physiologie der pflanzlichen Organisation, I. Teil, p. 12 angegebenen Methode mit Kaliumbichromatlösung injiziert. 
Trichome, die aus je einer Epidermzelle als Initiale hervorgehen.

Die Entwicklung des Nektariums beginnt, von der Mitte gegen den Rand des späteren Drüsenkomplexes fortschreitend, zu der Zeit, wenn die primären Gefäße im Blattpolster bereits deutlich differenziert sind. Zunächst vergrößern sich die Epidermzellen, wölben sich nach außen vor (Fig. $3, t^{1}$ ) und werden bald durch zwei senkrecht aufeinander orientierte Radialwände in Quadranten zerlegt (Fig. 3, t² Fig. 4). Dann teilt sich etwa in der Höhe der angrenzenden Epidermiszellen fast gleichzeitig jede dieser vier prismatischen Zellen durch eine tangentiale Querwand in zwei ungefähr gleiche Tochterzellen (Fig 4 und 5). Die untere der beiden so entstandenen Zellschichten bildet weiter keine Tangentialwände mehr aus und wird der spätere Fuß des Trichoms. Die oberen über das Niveau der angrenzenden Epidermiszellen vorgewölbten Zellen teilen sich jedoch bald von neuen tangential wiederum in je zwei Zellen, die ungefähr gleichgroß sind (Fig. 5 und 6). Nachdem diese zwölf Abkömmlinge der einen Epidermiszelle hiermit über die erste Phase ihres Jugendzustandes hinausgekommen sind, beginnen sie von nun ab, je nach den spezifischen Leistungen, die sie als Teile des fertigen Zellkomplexes zu erfüllen haben, sich zu modifizieren. In der Regel finden im weiteren Entwicklungsverlauf nur noch radiale Teilungen statt, öfter anfangs noch ziemlich gleichzeitig (Fig, 7) in den einzelnen Zelllagen. Im Fuß und Stiel schreitet beim ferneren Wachstum die Zellvermehrung korrespondierend fort. Die Basalzellen erreichen zuerst ihre definitive Gestalt, die Stielzellen nehmen an wenigsten an der Weiterentwicklung teil und werden insofern verändert, als nach vollendeter Zellteilung des Trichoms ihre Radialwände unter „Verkorkung“" verdickt werden. Die größte Umgestaltung erfährt die äußerste Zellschicht, deren Elemente sich unter sehr schnell aufeinander folgenden Radialteilungen zu dem sezernierenden Palissadengewebe differenzieren.

Die jugendliche Drüse weicht dadurch von der sezernierenden ab, daß sie über die Epidermis hervorragt, und daß ihre einzelnen Trichome höher und gestreckter erscheinen und dicht aneinander grenzen (Fig. 7). Wenn sich jedoch das Internodium und der Blattstiel zu strecken beginnen, dann werden bei der hierdurch erfolgenden Oberflächenvergrößerung die einzelnen Drüsentrichome etwas auseinander gerückt. Dadurch bekommen dic bis dahin eng aneinander gepreßten Köpfchen, die ihre definitive Höhe bereits erreicht haben, mehr Platz, dehnen sich infolgedessen - abgesehen von einer durch noch etwaiges aktives Wachstum erfolgenden Volumzunalıme - in die Breite aus und erscheinen somit in der ausgrebildeten Drüse platter als zuvor. Während auch die Basalzellen durch das Längenwachstum des Stengels etwas gestreckt werden, behalten die Stielzellen im großen und ganzen ihre Dimensionen, da ihre Radialwände bereits verdickt und "verkorkt" sind, wenn die einzelnen Trichome noch dicht zusammengedrängt stehen und die angrenzende Epidermis 
etwas überragen. Auf diese Weise ist das Entstehen der Zwischenräume zwischen den einzelnen Trichomen $\mathrm{zu}$ erklären.

Von der eben geschilderten Entwicklung gibt es natürlich oft mehr oder weniger erhebliche Abweichungen. So kommen statt der vier durch die primäre Radialteilung der Epidermzelle entstehenden Zellen bisweilen deren fünf vor; nicht selten eilt ferner nach der Ausbildung der drei Zellschichten die obere Zelllage den anderen gregenüber in der Bildung von Radialwänden voraus (Fig. 6), sodaß der spätere sezernierende Köpfchenteil bereits aus sechzehn Zellen besteht, wenn in den beiden darunter liegenden Zellschichten die sekundäre Radialteilung noch nicht begonnen hat. Öfter teilen sich auch die Köpfchenzellen tangential in zwei Palissadenschichten, und schließlich kommt es in letzterem Falle auch manchmal vor, daß die untere dieser beiden Schichten nicht aus prisnatisch gestreckten, sondern aus isodiametrischen Zellen besteht.

An der Ober- und Unterseite der Lamina, der Blattmittelrippe u. s. w. finden sich häufig einzelstehende Drüsenhaare von im wesentlichen gleichem Bau wie die Trichome des Nektariums: Fußteil, Stielzellen mit verdickten und verkorkten Radialwänden und prismatische Köpfchenzellen. Diese Trichome sind bereits vollständig ausgebildet, wenn das Nektarium erst angelegt wird.

\section{Polygonum baldschuanicum Regel. - Bokhara.}

Das extraflorale Nektarium befindet sich an der Unterseite des Blattpolsters als eine grubenförmige T'ertiefung, die bis $1 / 2 \mathrm{~mm}$ breit und $1 \mathrm{~mm}$ lang wird, meist jedoch bedeutend kleiner bleibt und oft nur gerade noch mit bloßem Auge zu erkennen ist. ${ }^{1}$ ) Die Sekretion ist im allgemeinen äußerst spärlich, und nur beim Austreiben im Mai und an warmen Tagen sah ich bisweilen eine Flüssigkeit ausgeschieden, die, wie die Reaktion mit Fehling'scher Lösung zeigte, eine reduzierende Zuckerart enthielt.

Soweit sich die grubige Einsenkung erstreckt, ist das Parenchym des Blattkissens, besonders die subepidermale Schicht etwas kleinzelliger als in der Umgebung. Die Zellen dieses Parenchyms stoßen ohne nennenswerte Interzellularen dicht aneinander, haben unregelnäßige, teils abgerundete, teils mehr polyedrische Form; im letzteren Fall sind sie plasmareich und stehen öfter in ununterbrochenem Zusammenhang mit den unter dem Nektarium liegenden Gefäßbündeln des Blattpolsters.

Das Nektarium wird auch in diesem Fall nur durch eine Gruppe - etwa fünfundzwanzig - dicht nebeneinanderstehender Trichome gebildet, ist also nichts weiter als eine lokale Anhäufung von Drüsenhaaren, die dadurch zustande kommt, daß sich an dieser Stelle jerle Epidermiszelle zu cinem 'Trichom umbildet.

I)ie einzclnen I)risenhaare werden durch mehr oder weniger

1) Das Nektarium ist nicht an jedem Blatt ausgebildet. 
große Zwischenräume (Fig. 8, l) getrennt und lassen je drei Teile erkennen.

Der Basalteil des Trichoms besteht aus drei bis vierzehn Zellen, die sich am Rande der Grube kontinuierlich in die umgebende Epidermis fortsetzen. Die Stielzellen sind meist in etwas geringerer Zahl als die Fußzellen vorhanden. Ihr Inhalt erscheint stark granuliert, farblos bis hellgelblich, ihre verdickten Radialwände werden mit Chlorzinkjod gelbbraun und bleiben auf mit Hämatoxylin gefärbten Schnitten farblos.

Das sezernierende Köpfchen besteht aus unregelmäßig gestalteten, teilweise prismatischen Zellen, die meist mehr oder weniger fächerförmig angeordnet sind (Fig. 8, k), ein dichtes Plasma und einen relativ großen Zellkern besitzen.

Wie ein mit Ferrichlorid behandeltes sezernierendes Nektarium zeigt, ist in den Trichomen und dem oben erwähnten „subglandularen" Parenchym viel Gerbstoff vorhanden.

\section{Einige andere Polygonaceen.}

Außer den beiden eben geschilderten Polygonaceen untersuchte ich näher noch die extrafloralen Nektarien von Polygonum Convolvulus Linn. (gemäßigte nördliche Zone), Polygonum dumetorum Linn. (gemäßigte nördliche Zone), Polygonum multiflorum Thunb. (China, Japan). Die Drüsen finden sich in allen diesen Fällen an gleicher Stelle wie bei Polygonum baldschuanicum. Die Entwicklungsgeschichte verfolgte ich bei Polygomm dumetorum und fand, daß sie von der bei Muehlenbcckia ausführlich beschriebenen nicht wesentlich abweicht.

Bei Polygonum dumetorum stehen die einzelnen Drüsenhaare meist weiter voneinander ab, als es bei Muehlenbeckia der Fall ist. Ihr sezernierendes Köpfehen, das dem von Polygonum baldschuanicum durch fächerartige Anordnung seiner Zellen ähnelt, durch palissadenartige Form der einzelnen Elemente jedoch davon abweicht, ist an der Basis verbreitert und greift oft helmartig über den Stielteil hinüber, sodaß man je nach der Schnitthöhe auf Flächenansichten in demselben Köpfchen oben etwa 30, unten etwa 50 Palissadenzellen sieht. Der Stielteil besteht aus drei bis zehn Zellen mit verdickten und verkorkten Radialwänden. Gerbstoff findet sich in gleicher Verbreitung wie bei Muehlenbeckia.

Polygonum Convolvulus zeigt keine Abweichungen von eben genannter Spezies.

Bei Polygonum multiflorum wird die sezernierende Fläche von fünfzehn bis zwanzig Trichomen gebildet, die in ihrem Bau denen von Muchlenbeckia ähneln, nur durch größere Zwischenräume getrennt werden und stärker plattgedrückt erscheinen, meist auch einen an der Basis verbreiterten Köpfchenteil erkennen lassen; Gerbstoff ist in gleicher Weise wie bei Muchlenbeckia verbreitet. 
Gossypium brasiliense Macfad. - Trop. Amerika.

a) Sezernierendes Nektarium.

An der Unterseite der Blattmittelrippe, bisweilen auch an entsprechenden Stellen einiger anderer stärkerer Rippen des handnervigen Blattes befindet sich etwa $8 \mathrm{~mm}$ von der Basis der Lamina entfernt eine grubige Vertiefung, die sehr verschieden geformt sein kann (elliptisch, kreisförmig, dreieckig, auch rinnenartig verlängert) und das extraflorale Nektarium darstellt. Im Bereich dieser Einsenkung ist die Blattmittelrippe oft etwas verbreitert. Die reichlich ausgeschiedene Flüssigkeit reagiert auf eine reduzierende Zuckerart, ist also Nektar.

Ein Querschnitt zeigt, soweit die Nektar ausscheidende Fläche sich erstreckt, halbkreisförmig darunterliegend ein Gewebe, das zwar in manchen Punkten, z. B. Kleinzelligkeit, von seiner Umgebung abweicht, aber nicht als typisches Drüsengewebe zu bezeichnen ist; es besteht gegen den Rand der Grube hin aus vier, in der Mitte aus etwa acht Schichten polygonaler Zellen, die, je näher der Epidermis, desto dichter aneinanderschließen. Unter diesem subglandularen Gewebe liegt die Gefäßbündelmasse der Blattmittelrippe durch einige Schichten größerer abgerundeter Parenchymzellen von ihm getrennt.

Der Grund der grubigen Vertiefung ist mit zahlreichen - etwa 240 - Drüsenhaaren bedeckt (Fig. 13, $t$ ), deren frei hervorragender Teil auf tangentialen Flächenschnitten einen elliptischen Umriß zeigt, und zwar in axialer Richtung gestreckt ist (Fig. 9), wodurch bewirkt wird, daß die Trichome auf Längsschnitten flacher und breiter, auf Querschnitten höher und schmaler erscheinen. Die einzelnen Drüsenhaare sind in Fuß, Stiel und Köpfchen gegliedert.

Die Basalzellen - etwa zehn bis fünfzehn in einem Trichom - wölben sich nach oben verjüngt sockelartig etwas hervor, sodaß die die einzelnen Drüsenhaare trennenden Zwischenräume als nach unten konisch zugespitzte Rille in prägnanten Fällen bis zur Mitte der Basalzellen einschneiden (Fig. 10, b). (Bei Vicia amphicarpa, V. Faba, V. sepium ist es ähnlich.) Damit hängt es zusammen, daß auf tangentialen Flächenschnitten, die gerade durch die tiefste Stelle dieser Einschnitte geführt werden, die Basalteile der einzelnen Trichome noch deutlich voneinander abgegrenzt erscheinen, und zwar wabenartig in Form axial gestreckter Sechsecke, die bisweilen kahnförmig zugespitzt sind (Fig. 11), dicht aneinanderstoßend.

Der Stiel besteht aus einer einzigen Zelle. Ihre stark verdickte Radialwand, die auf mit Hämatoxylin tingierten Präparaten farblos bleibt, wird mit Chlorzinkjod gelbbraun und speichert mit Fuchsin gefärbt diesen Farbstoff sehr intensiv. In jüngeren Stadien ist die Wand noch unverdickt und färbt sich mit Hämatoxylin blau; sie wird erst verdickt und chemisch verändert, wenn der Entwicklungsgang des Nektariums abgeschlossen ist. Meist sieht man diese Wandverdickung sich auch auf die an die freie Oberfläche grenzende Membran der Fußzellen fortsetzen (Fig. 10, w). 
Die Cuticula ist als dünnes Häutchen mehr oder weniger deutlich von ihr abgesetzt, also ihr stofflich nicht vollständig gleich. An älteren Nektarien bemerkte ich bisweilen auch in der Mitte der unteren Tangentialwand des Stieles eine scheibenförmige verdickte Stelle, vielleicht ein Zeichen für das beginnende Erlöschen der Sekretion.

Das Köpfchen endigt ellipsoidisch abgerundet und besteht aus etwa sechzig bis hundert Zellen -- das einzelne Trichom ist also im Mittel aus ungefähr neunzig Zellen zusammengesetzt -, die, wie man öfter konstatieren kann, ihrer Entwicklung gemäß in (vier) Schichten angeordnet sind. Diese sezernierenden Zellen sind plasnareich, und besitzen äußerst zarte Membranen. Die Cuticula geht kontinuierlich über die ganze Oberfläche der Trichome hinweg und erscheint über den Köpfchen nicht selten abgehoben.

In den einzelnen Teilen der Trichome und den zwei hypodermalen Zellschichten sind gelbbraune Massen enthalten, die durch Alkohol nicht ausgezogen wurden (die Objekte waren vorher mit Formalin behandelt) und auch bei wochenlangem Verweilen in Xylol sich nicht lösten. Ich möchte diese Inhaltsstoffe, zumal auch durch längeres Einwirken von Eisenchlorid an gleichen Stellen eine Schwarzfärbung entsteht, als gerbstoffhaltig ansprechen. ${ }^{1}$ ) Enorm reich an diesem Inhalt ist speziell der Köpfchenteil der Trichome. In dem subglandularen Gewebe kommen zerstreut Drusen von Calciumoxalat vor.

Den nektarausscheidenden Trichomen im wesentlichen gleich gebaute, denselben Inhalt enthaltende Drüsenhaare finden sich auf der Blattoberseite und den Seiten der Blattmittelrippe. Ihre Entwicklung ist auch in diesem Falle bereits abgeschlossen, wenn die des Nektariums beginnt.

\section{b) Entwicklungsgeschichte.}

Dienektarabsondernden Gebilde lassen sich genetisch auf eine Epidermiszelle zurückführen, sind also Trichome.

Ihre Entwicklung beginnt verhältnismäßig spät, nämlich wenn die Gefäßbündel der Blattmittelrippe bereits deutlich differenziert sind; sie schreitet von der Mitte einer ganz flachen Einsenkung ausgehend gegen die Peripherie hin fort.

Zunächst (Fig. 12) wölbt sich eine Epidermzelle über das umgebende Niveau papillenartig weit nach außen vor und bildet alsclann, wenn sie etwa doppelt so lang als breit ist, ungefähr in der Höhe der Außenfläche der angrenzenden Epidermis, eine tangentiale Querwand aus. Die untere der beiden so entstandenen Zellen teilt sich im ferneren Entwickiungsgange nur noch senkrecht zur Oberfläche und wird zum Fuß, der sich später durch akropetales Wachstum etwas nach anßen vorstreckt. Die obere \%elle greht schnell hintereinander weitere Querteilungen cin

1) cf. Zacharias, Über Sekretbehälter mit verkorkten Membranen. Bot. Ztg. 1879 . p. 621 . 
und besteht so successive aus zwei, drei, vier, fünf etagenartig iibereinanderliegenden Zellen. Die unterste dieser fünf Zellen teilt sich nicht weiter und ist die spätere Stielzelle; aus den oberen vier Zellen geht durch lebhafte Radialteilungen das spätere Köpfchen hervor.

Ich brauche wohl kaum hervorzuheben, daß es Ausnahmen von der Zahl der einzelnen Zellschichten gibt, daß zum Beispiel das Köpfchen manchmal aus drei oder aus fünf Etagen besteht.

\section{Einige weitere Gossypien.}

Außer vorstehender Spezies wurden noch die Nektarien von Gossypium Davidsonii Kellogg. (Westl. Nordamerika), Gossypium herbaceum Linn. (Trop. Asien), Gossypium microcarpum Tod. (Mexiko) untersucht. Bei allen diesen Arten ähneln sich die Drüsen außerordentlich und lassen keine erheblichen Abweichungen von Gossypium brasiliense erkennen. Wie bei den einzelnen Individuen die Gestalt des ganzen Nektariums sehr variiert, besonders durch mannigfache Emergenzen oft das verschiedenste Aussehen erhält (Fig. 13 em), so kann auch die Form der Trichome bei derselben Spezies wechseln. Im folgenden seien einige kleinere, jedoch keineswegs immer vorhandene, und demnach natürlich durchaus nicht durchgreifende Unterschiede gegen Gossypium brasiliense genannt.

Die Trichome von Gossypium Davidsonii sind meistens kleiner und besitzen im allgemeinen weniger Basalzellen, in der Regel etwa drei, bisweilen auch nur eine.

Bei Gossypium herbaceum stehen auf der gleichen Fläche verhältnismäßig mehr Drüsenhaare; sie haben demgemäß meist polygonal gestaltete Köpfchen, die, wie besonders Flächenschnitte erkennen lassen, dicht aneinander grenzen.

Bei allen diesen Arten ist die Anordnung der Köpfchenzellen in drei bis vier Etagen bisweilen deutlich ausgeprägt. Man darf somit bei allen - für Gossypium herbaceum kann ich es bestätigen auf die gleiche Entwicklung schließen, wie sie für Gossypium brasiliense angegeben wurde.

\section{Tecoma radicans Juss. - Nordamerika.}

Aus der Literatur sind mir zwei Angaben über die Anatomie der bei obengenannter Pflanze vorkommenden extrafloralen Nektarien bekannt geworden, die eine von Poulsen ${ }^{1}$, die andere von Morini ${ }^{2}$ ) herrührend. Da jedoch beide Autoren manche wesentlichen Gesichtspunkte nicht in Betracht gezogen haben und beide nur eine recht knapp gehaltene Beschreibung geben, so will ich meine eigenen Untersuchungen im folgenden mitteilen.

Die von mir studierten extrafloralen Nektarien - nach Delpin $0^{3}$ ) hat diese Pflanze fünferlei nektarabsondernde Drüsen finden sich in größerer Zahl (bis etwa Zwanzig) auf der Oberseite

1) l. c. p. 261 .

2) 1. c. p. 355 .

3) Zitiert nach Just. Bot. Jahresber. 1902, p. 443. 
des Blattstieles und zwar im unteren Viertel desselben da, wo die beiden Flügel leistenartig an ihm herablaufen. Sie erscheinen mit bloßem Auge betrachtet als kleine in der Richtung des Blattstieles verlängerte Grübchen, die von einem etwas hervorragenden Gewebewulst rings umgeben werden.

An warmen Tagen fand ich öfter, besonders reichlich beim Austreiben der Pflanze, an den eben genannten Stellen kleine Tröpfchen ausgeschieden, die mit Fehlings Lösung zum Sieden erhitzt Kupferoxydul ausfällten.

\section{a) Sezernierendes Nektarium.}

Ein Schnitt durch ein solches Grübchen zeigt am Grunde einer flachen Einsenkung ein napfförmig gestaltetes Gebilde, das passend mit einer Trinkschale zu vergleichen ist, die auf zwei Seiten etwas zusammengepreßt, also ellipsoidisch begrenzt ist; diese Schüssel ist als sezernierender Teil eines einzigen Trichoms aufzufassen.

Die Fußzellen des Drüsenhaares sind in flach konkaver Schicht angeordnet und weichen von der angrenzenden Epidermis, deren Fortsetzung sie bilden, durch ihr geringeres Volumen ab.

Auf diese Basalschicht folgen die Stielzellen - etwa achtzig bis neunzig -, meist kubisch geformt. Ihre Radialwände sind stark verdickt und werden mit Chlorzinkjod gelbbraun; letzteres ist auch manchmal an einigen Tangentialwänden der Fall. Auf mit Hämatoxylin gefärbten Schnitten bleibt diese Schicht farblos. Das nach außen daranschließende Köpfchen oder der sezernierende Teil besteht in der Regel aus einer Schicht sehr zartwandiger, ungemein zahlreicher - zweitausend ist wohl nicht zu viel gesagt Palissadenzellen, deren Anordnung an den verschiedenen Stellen der Schüssel wechselt. Sie sind im großen und ganzen radial ungefähr gegen die Mitte des schüsselförmigen Hohlraums hin geordnet. Soweit sie direkt auf den Stielzellen fußen, sind sie senkrecht zur Oberfläche der konkaven Basalschicht orientiert, mit Ausnahme der drei äußersten peripheren an die inneren Seitenwände der Grube grenzenden Schichten, die auf Schnitten entweder eine fächerartige Ausbiegung nach außen erkennen lassen oder keilförmig eingesetzt erscheinen und zwar mit der Spitze auf den Hohlraum zu gerichtet (Fig. 14, $k_{1}, k_{2}$ ).

Über dieser letzteren Zone liegt der nach innen etwas übergewölbte periphere Außenrand der Schale, etwa drei bis acht Zelllagen umfassend, deren unterste Schicht tangential gerichtete Längswände zeigt, während die übrigen Zellen, je weiter nach außen, desto mehr nach unten gerichtet sind und gleichzeitig kürzer werden (Fig. 14, r).

Über das Trichom geht eine derbe Cuticula hinweg, die

besonders tief am Außenrand des Köpfchens - zahnartig zwischen die einzelnen Palissadenzellen eingreift ${ }^{1}$ ) und in der Mitte des Köpfchens oft blasenartig emporgehoben erscheint.

1) Das gleiche ist bei der sezernierenden Oberfläche der Nektarien von Acacia lophantha und Turnera angustifolia der Fall. 
An den Fuß des eben geschilderten Haargebildes schließt nach innen zu ein Gewebe an, dessen Zellen in mancher Beziehung von ihrer Umgebung abweichen, jedoch weniger durch Form und Inhalt als durch ihre Anordnung. Den Ausdruck "Drüsengewebe“ möchte ich auch in diesem Falle vermeiden, und nenne es daher einfach zuleitendes Gewebe (Fig. 14, $h$ ). Die wahre Gestalt dieses Gewebes ist nur aus der Kombination von Quer-, Längs- und Flächenschnitten zu ersehen.

Unter dem Trichom liegen etwa vier Zellschichten, deren Elemente durch etwas dichteren Inhalt, geringeres Volumen und lückenloses Aneinanderschließen von ihrer Umgebung abweichen. Die an den Außenrand des Fußes ansetzenden Zellen erscheinen auf Querschnitten radial auf das Trichom angeordnet; auch in der Längsrichtung sind die Rindenzellen des Blattstieles etwa ein- bis zweimal so weit, als der Fuß des Trichoms reicht, auf die Drüse $\mathrm{zu}$ gestreckt. An dieses zuleitende Gewebe gehen reichlich Gefäßbündelendigungen heran, die zum größten Teil Abzweigungen der beiden außerhalb des zentralen Bündelringes liegenden Stränge der Flügel sind, manchmal aber auch aus dem zylindrischen Zentralring selbst entspringen.

Wird ein Blattstiel, dessen Nektarien sezernieren, mit Kaliumbichromat injiziert, so lassen nicht allein die einzelnen Teile des Trichoms einen reichen Gerbstoffgehalt erkennen, sondern auch das zuleitende Gewebe hebt sich, strahlenförmig vom Fuß der Schüssel ausgehend, durch die in ihm reichlich vorhandenen, dunkel ausgefällten Gerbstoffmassen von der an Gerbstoff weniger reichen Umgebung ab.

Der Bau des Trichoms kann in Einzelheiten beträchtlich variieren: Es kommen zum Beispiel nicht nur öfter zwei Stielschichten, zwei Palissadenschichten, sondern auch nicht selten Übergänge vor in der Weise, daß diese zwischen Stiel und Köpfchen liegende dritte Schicht zum teil aus Palissaden-, zum teil aus isodiametrischen Zellen mit verdickten Radialwänden besteht.

b) Entwicklungsgeschichte.

In morphologisch-entwicklungsgeschichtlicher Hinsicht ist das Nektarium ein epidermales Gebilde, ein Trichom.

Die ersten Stadien sind insofern nicht ganz leicht zu finden. als der junge Blattstiel an gleichen Stellen mit anderen Drüsenhaaren dicht besetzt ist, die den jungen Zuständen des Nektariums. täuschend ähneln. Diese kleineren Trichome entwickeln sich sehr früh und sind z. t. schon ausgebildet, wenn die Nektarien sich anzulegen beginnen; sie finden sich sehr zahlreich auch an der jungen Sproßachse, der Blattober- und -unterseite.

Die Entwicklung des Nektariums wird dadurch eingeleitet, daß eine Epidermzelle sich vorwölbt und dann bald in drei Teile gliedert, den Fuß, den Stiel und das kugelförmig darauf sitzende Köpfchen. Durch lebhafte Radialteilungen erreichen die einzelnen Teile 
allmählich ihre definitive Gestalt und Größe. Das Entstehen des äußeren oft nach innen übergreifenden Randes des sezernierenden Köpfchens dürfte auf ein hier vorhandenes stärkeres Wachstum zurückzuführen sein. Die Radialwände der Stielzellen bleiben mit Hämatoxylin schon farblos - die Zeit kann jedoch sehr variieren -, wenn das zuleitende Gewebe als solches noch nicht differenziert ist. In diesem Stadium sind die primären Gefäße im zentralen Ring des Petiolus bereits angelegt, an den Stellen der späteren zuführenden Gefäßbündel befinden sich noch zarte Prokambien, und die Drüse selbst ragt als ein in der Mitte vertieftes Näpfchen über die angrenzende Epidermis hervor.

Daß sie nach abgeschlossenem Entwicklungsgang in einer grubigen Vertiefung liegt, scheint mir ebenso leicht mechanisch erklärt werden zu können, als die spätere radiale Anordnung des Zuleitungsgewebes: nämlich meines Erachtens kommit die radiäre Streckung vor allem beim Längenwachstum des Blattstieles, die Einsenkung dagegen beim gleichzeitigen Dickenwachstum des Petiolus dadurch zustande, daß di e Zellen im Bereich des Nektariums sich passiv verhalten, indem sie klein bleiben und sich am allgemeinen Wachstum nicht beteiligen.

\section{Ligustrum Regelianum Hort. Lemoine. - Japan.}

a) Ausgebildetes Nektarium.

Die untersuchten Nektarien finden sich in größerer Anzahl - etwa zehn - auf der Blattunterseite und können sich, wenn sie dicht an der Mittelrippe liegen, öfter auch ein wenig auf letztere hin ausdehnen. Sie sind an der Basis der Lamina nicht selten dicht nebeneinander angehäuft und erscheinen im auffallenden Licht betrachtet als dunkelgrüne Punkte, oft glänzend durch das ausgeschiedene Sekret, das sich als zuckerlaltig erwies.

Das Nektarium liegt in einer flachen Einsenkung, der eine geringe Erhebung der Blattoberseite entspricht und besteht aus einem epidermalen sezernierenden Teil und einem darunter liegenden wohl differenzierten Drüsengewebe (Fig. 19).

Die Epidermis der Blattunterseite ist, soweit sich die flache Einsenkung erstreckt, in mehr oder minder zahlreiche - die Anzahl variiert beträchtlich, ich zählte fünfzehn bis sechzig 'T'richome meist ungemein dicht nebeneinanderstehende Drüsenhaare umgestaltet, an denen man, wie in allen übrigen von mir untersuchten Fällen mit dergleichen Gebilden Fuß, Stiel und Kopf unterscheiden kann. Säntliche Teile des einzelnen Trichoms sind reich an Plasna und enthalten einen relativ großen Kern. Die Epidermis der Blattunterseite ist am Rande des Nektariums senkrecht zur Blattfläche gestreckt bezw. zweischichtig und setzt sich in die Fußzellen der Drüse fort.

Bisweilen ist der aus drei bis fünf Zellen bestehende Basalteil des einzelnen Trichoms als solcher deutlich dadurch abge- 
grenzt, daß er sich etwas hervorwölbt und so einen Sockel für die auf ihm sitzende Stielzelle bildet.

Letztere ist in der Regel in der Einzahl vorhanden und in älteren Stadien auf den ersten Blick auffallend durch eine eigentümliche Verdickung ihrer Radialwände ${ }^{1}$ ) (Fig. 15, r). Vom Köpfchen an gerechnet nimmt die Membran zunächst allmählich an Dicke zu, springt dann bogenförmig in das Innere der Stielzelle vor, so einen Ringwulst bildend, der das Zelllumen beträchtlich einengt, und wird schließlich unmittelbar über der inneren Tangentialwand plötzlich wieder dünner ${ }^{2}$ ). Diese Verdickung kann sich auch auf die obere Außenseite der Fußzellen fortsetzen, ja zuweilen sogar zwischen die Basalteile der einzelnen Trichome zahnförmig eingreifen.

Stehen die Drüsenhaare, wie es fast stets der Fall ist, sehr dicht nebeneinander, so zeigen die beiden Verdickungsringe je zweier Stielzellen auf Querschnitten ein herzförmig erscheinendes Bild (Fig. 15); sind die einzelnen Trichome dagegen auseinandergerückt, so sieht man über die zwischen ihnen liegenden Basalzellen diese Verdickung merklich verdünnt, wie eine recht derbe Cuticula hinweggehen. Bei starker Vergrößerung - ich beobachtete es an mit Anilinblau tingierten Objekten ist die Cuticula, die als zartes Häutchen das ganze Trichom bedeckt, auch außerhalb dieser Membran zu erkennen; demnach dürfte diese Radialwandverdickung, die mit Chlorzinkjod eine braune Färbung annimmt, stofflich mit der Cuticula nicht identisch sein.

Sind in einem Trichom zwei oder drei Stielzellen vorhanden, so ist ihre gemeinsame Radialwand bisweilen auch "verkorkt" und sehr schwach verdickt, jedoch meist gleichmäßig ohne den wulstförmigen Ring (cf. Fig. 16,s). Auf mit Hämatoxylin tingierten Schnitten bleibt die Radialwand farblos. Auf Querschnitten erscheint die Stielzelle konisch zugespitzt, in der Flächenansicht elliptisch; sie hat demnach räumlich die Gestalt eines mehr oder weniger deformierten an der Spitze meist abgerundeten Kegels.

Das sezernierende Köpfchen (Fig. 15, k, Fig. 17) wird von etwa 15 prismatischen Zellen gebildet, die in der Mitte kürzer sind als am Rande des Trichoms, wo sie oft helmartig über die Stielzelle hinübergreifen.

Soweit diese Sekretionsfläche reicht, ist an Stelle des sonst sehr lockeren Schwammparenchyms des Blattes ein Drüsengewebe vorhanden, das durch dichtes Aneinanderschließen und Plasmareichtum seiner Zellen wohl charakterisiert ist; die Elemente dieses Nektariumgewebes sind isodiametrisch, haben geringes Volumen und relativ großen Kern und gehen allmählich in das benachbarte Blattmesophyll über. An die dem Palissadengewebe der Blattoberseite zu gelegenen, durch kleine Intercellularen getrennten Drüsen-

1) Diese Verdickung ist auch bei den am Rande stehenden Trichomen in gleicher Weise ausgebildet. Morini, (l. c.), der sie für Ligustrum lucidum in seinen Figuren hier nicht angibt, dürfte sich geirrt haben.

${ }^{2}$ ) Letzteres braucht nicht immer der Fall zu sein (Fig. 15). 
zellen gehen Gefäßbündelendigungen heran. Das über dem Nektariumgewebe liegende Palissadenparenchym ist konzentrisch auf die Sekretionsfläche zu gerichtet. Diese Anordnung, die für die Stoffleitung sehr zweckmäßig sein mag, ist auf ein Nichtteilnehmen des Drüsengewebes am Flächenwachstum des Blattes zurückzuführen.

b) Entwicklung.

Die Entwicklungsgeschichte lehrt, daß die einzelnen sezernierenden Gebilde Trichome sind, die aus je einer Epidermzelle entstehen.

Die Anlage der Drüsenhaare beginnt, wenn das Blatt noch durchaus meristematischen Charakter hat und das spätere Palissadengewebe von dem späteren Schwammparenchym noch nicht zu unterscheiden ist.

Eine Epidermzelle wölbt sich zunächst nach außen vor, teilt sich dann tangential in 2 Zellen, deren äußere die Teilung bald wiederholt, so daß nunmehr 3 übereinander liegende Zellen vorhanden sind (Fig. 18, Fig. 19). Durch Radialteilungen wird dann allmählich die äußere Zelle zum Köpfchen, die innere zum Fuß, während die mittlere gewöhnlich ungeteilt bleibt. Wenn nach Abschluß der Teilungen der Aufbau des Trichoms soweit vollendet ist, daß nunmehr die Feinheiten der Struktur ausgebildet werden, dann erzeugt die Stielzelle in kurzer Zeit die erwähnte interessante Wandverdickung.

Das unter der Sekretionsfläche liegende Drüsengewebe ist in jungen Stadien durch nichts, wenigstens in anatomischer Hinsicht, von dem umgebenden noch meristematischen Gewebe unterschieden und hebt sich erst später von dem Mesophyll dadurch ab, daß es im großen und ganzen auf dem während des jugendlichen Zustandes eingenommenen Umfang verharrt, ohne sich am Flächenwachstum aktiv zu beteiligen.

Auf beiden Seiten der ausgebildeten Lamina stehen vereinzelt - angelegt werden bedeutend mehr solche Trichome, jedoch zum größten Teil abgestoßen, sobald das junge Blatt zu assimilieren beginnt - in kleine Grübchen eingesenkte Drüsenhaare, die dieselbe Struktur haben wie die einzelnen Trichome des Nektariums, und zwar bis auf Einzelheiten ihnen täuschend ähneln, indem z. B. ihre Stielzelle gleichfalls die charakteristische Wandverdickung besitzt und mit Hämatoxylin farblos bleibt (Fig. 20). Ihr Köpfchen ist meist etwas kleiner und besteht aus etwa 8 Zellen, ihre Entwicklung, die zum Teil schon abgeschlossen ist, wenn das Nektarium sich anzulegen beginnt, stimmt mit der der nektarabsondernden Drüsenhaare überein, auch in der Hinsicht, daß in beiden Fällen die Trichome in jüngeren Stadien über die Epidermis hervorragen.

Die von mir außerdem untersuchten Nektarien von Ligustrum vulgare Linn. (Europa), Syringa chinensis Willd. (China), Syringa persica Linn. (Persien) zeigen keine erheblichen Abweichungen von dem vorstehend geschilderten Typus. 


\section{Polypodiaceen.}

Die im folgenden genannten Farne sind nach Goebeli), dem wir die genaue Kenntnis ihrer biologisch interessanten Einrichtungen verdanken, sämtlich humussammelnde Epiphyten. Die Nektarien einiger Arten sind außer von Goebel2) auch von Beccaris) erwähnt, der ihnen die Rolle zuschreibt, Ameisen anzulocken.

An den ersten Blättern bildeten die im Göttinger botanischen Garten mir zur Verfügung stehenden kultivierten Exemplare keine Nektarien aus. Die Angabe Beccarist): „Sulle fronde sterili delle varie specie di Polypodium, affini al quercifolium, non ho osservato alcuno accenno di nettarii, analoghi a quelli esistenti nel Polypodium nectariferum" ist mir unverständlich; soweit hier Spezies mit dimorphem Laub in Kultur sind, haben auch die Nischenblätter (fronde sterili) Nektarien.

\section{Drynaria Linnaei Bory. - Altweltliche Tropen.}

Da das hier kultivierte Exemplar während meiner Untersuchungen keine Nischenblätter bildete, so untersuchte ich nur die Nektarien der Fiederblätter. Die Nektarien sind am auffallendsten in den spitzen Winkeln zwischen der Blattmittelrippe und den Seitennerven erster Ordnung, wo sie bis $3 \mathrm{mnl}$ Durchmesser erreichen und als dunkelgrüne Flecken mit hellem Hof erscheinen; je nach der Größe des Blattes variiert auch die Zahl der Nektarien; ich zählte öfter ungefähr 20 derartige große Drüsen auf einem Blatt; außerden sind auch sonst auf der Spreite reichlich Nektarien vorhanden, nur bedeutend kleiner, im übrigen aber gleich gebaut, wie die eben erwähnten größeren Drïsen.

Die ausgeschiedene süß schmeckende Flüssigkeit enthält, wie die Reaktion mit Fehling'scher Lösung zeigte, eine reduzierende Zuckerart. Die Sekretion war in den Göttinger Gewächshäusern von Anfang an sehr reichlich. Sobald das eingerollte Blatt sich an der Basis zu entfalten beginnt, sondern die an den herablaufenden Flïgeln befindlichen Nektarien große Tropfen aus. Mit dem Heranwachsen des Blattes nimmt die Zahl der sezernierenden Drüsen zu; icl habe nie eine so reichliche und so lange dauernde Sekretion bei extrafloralen Nektarien gesehen, wie bei den hier genannten Polypodiaceen. So schieden an einem 14 Tage alten Blatt 10 Nektarien Flüssigkeit aus, und etwa 10 Tage später sezernierten auf demselben Fiederblatt gleichzeitig 15 größere und 25 kleinere Drüsen, und zwar kann ein und dasselbe Nektarium sowohl auf der Oberseite, als auch auf der Unterseite der Lamina Nektar ausscleiden. Dieser für extraflorale Nektarien bis jetzt nicht bekannte Typus findet seine Erklärung in den anatomischen Verhältnissen der Drüse.

1) Go ebel, Pflanzenbiologische Schilderungen I, p. $214 \mathrm{ff}$.

2) l. c. p. 219.

3) Beccari, Malesia p. $243 \mathrm{ff}$.

${ }^{4}$ Beccari, l. c. p. 246. 
Auf einer Flächenansicht zeigt die Epidermis des Blattes in der Ungebung des Nektariums mancherlei Veränderungen: während die normalen Epidermzellen wellenartig gebogene Seitenwände haben, werden die Zellen, je näher der Drüse, desto geradwandiger und kleiner und lassen deutlich eine konzentrisch auf das Nektarium zu gerichtete Anordnung erkennen (Fig. 21, ep). Die Epidermzellen des Nektariums selbst sind klein und geradwandig. Auf der Blattunterseite nehmen die Spaltöffnungen nach dem Nektarium zu an Zahl ab, und statt ihrer sind in mehreren Zellen hufeisenförmige Antiklinen zu erkennen, die ich als die ersten Anlagen von Spaltöffnungen auffasse, was durch die Entwicklungsgeschichte zu beweisen sein wïrle; letztere habe ich in anbetracht des wertvollen Materials nicht untersucht, glaube sie jedoch voraussagen zu können.

An jungen Blättern sieht man die Epidermis genau so beschaffen, wie über dem sezernierenden Nektarium, nämlich kleinzellig, geradwandig, und als erste Anlage der Spaltöffnungen jene halbkreisförmigen Wände.

Somit habe ich allen Grund, anzunehmen, daß auch in diesem Fall das Nektariumgewebe auf jenem meristematischen $\mathrm{Zu}$ stand verharren blieb, ohne weitere Zellteilungen einzugehen, während das Blatt weiter wuchs; durch ein derartiges Verhalten muß dann die erwähnte radiale Anordnung und Streckung der Zellen zustande kommen.

Die wenigen auf dem Nektarium befindlichen Spaltöffnungen sind anatomisch kaum von den luftatmenden verschieden; ihre Funktion jedoch dürfte eine andere sein, nämlich die der Nektarabsonderung (,Saftrentile“ Behrens' 1).) An der Oberseite des Nektariums, die im allgemeinen weniger reichlich sezerniert, sind keine besonderen Austrittsöffnungen vorhanden; das Sekret dürfte in diesem Fall, wie es auch für die Wasserapparate einiger Farne und für nicht wenige Nektarien bekannt ist, die "kutikularisierte“ Vembran passieren.

Das Nektariumgewebe, das allmählich in das angrenzende Blattparenchym übergeht, durchsetzt, wie ein Querschnitt zeigt, das Blatt gleichmäßig und besteht aus kleinen, dünmwandigen, plasmareichen Zellen, die nur geringe Interzellularen zwischen sich lassen (bedeutend kleiner, als es im Nektarium von Pteris aquilina der Fall ist, ef. Fig. 22, $n$ ). An das Drüsengewebe gehen Gefäßbündelendigungen heran (Fig. 23,g).

Wie ein auf dem Stadium der Sekretion mit Kaliumbichromat behandeltes Blatt erkennen läßt, ist das Drüsengewebe enorm reich an Gerbstoff, der hier in Ballen kleiner, dunkler Kugeln niedergeschlagen ist - in gleicher Weise in der Stärkescheide der Gefäßbündel ‥ und dem Nektarium so ein dunkleres Aussehen gibt; in den anstokenden Blattgewebe ist der Gerbstoff mehr gleichmäßig hell ausgefällt.

Eisenchlorid färlot das Drüisengewebe blauschwarz. Stärke

1) Behrens, J. W., Die Nektarien der Blüten. Flora 1879. 
fehlt im Nektarium, während sie sonst im Blatt reichlich vorhanden ist.

Drynaria quercifolia Bory. - Paläotrop. Region.

Diese Pflanze war nach Beccari1) schon den alten Autoren bekannt; über ihre Nischenblätter schreibt bereits Rumphius²) 1750: „radicem adeo obtegunt, ut vix dignosci possit, ipsorumque concava pars plerumque formicis repleta est."

Die Nektarien dieser Art sind denen von Drynaria Limnaei gleich gebaut; sie kommen hier auch an denselben Stellen vor, wie bei Drynaria Linnaei, und außerdem findet sich meist noch in dem stumpfen Winkel zwischen der Blattmittelrippe und den Seitennerven erster Ordnung je 1 größeres Nektarium, sodaß der einzelne Fiederabschnitt 2 große und daneben noch etwa 12 kleinere Nektarien hat; an einem $75 \mathrm{~cm}$ langen Fiederblatt zählte ich 47 große und etwa 250 kleinere Nektarien; an einem 3 Wochen alten Blatt sonderten gleichzeitig 3 große Nektarien an der Unterseite und 11 kleinere an der Oberseite Nektar ab.

Außer bei diesen beiden Spezies schieden in den Göttinger Gewächshäusern die Nektarien von Drynaria rigidula J. Sm. und Polypodium Meyeniamum Schott ${ }^{3}$ ) Nektar aus. Morphologisch gleiche Drüsen, die höchst wahrscheinlich auch Nektarien sind, fand ich außerdem bei Polypodium Heracleum Kze. und Polypodium coronans Wall. Bei allen diesen Arten sind die Nektarien ziemlich gleich gebaut; bei Polypodium Heracleum und Polypodium Meyenianum liegen die großen Drüsen hauptsächlich in dem stumpfen Winkel zwischen Blattmittelrippe und den Seitenrippen.

\section{Acacia cornigera Willd. - Mexiko.}

Diese für den Biologen so äußerst interessante Pflanze - von Delpin $0^{4}$ ) eine „specie formicarie piu segnalate“ genannt — ist in der Literatur schon öfter erwähnt und von Belt, Fritz Müller, Schimper ${ }^{5}$ ) u. a. näher studiert worden. Ihr extraflorales Nektarium jedoch hat merkwürdigerweise, soweit ich ermitteln konnte, bis jetzt noch keine anatomische Bearbeitung erfahren.

Morphologisch ist es längst bekannt und gehört zu den weitverbreiteten, oft zur Charakteristik benutzten „glandulae petiolares" der alten Systematiker. Die Drüse befindet sich an der Oberseite des Blattstieles, etwa $3 \mathrm{~mm}$ über der Ansatzstelle der beiden bekannten Stipulardornen beginnend, und hat die Gestalt eines empor-

\footnotetext{
1) Beccari, l. c. p. 246.

2) ibidem.
}

3) B e c cari, l. c. p. 247 sagt betr. Polypodium Meyenianum und Polypodium Heracleum: "Non ho osservato nettarii sopra le fronde delle due specie citate", was ich mir nur damit erklären kann, daß er die Drüsen übersehen haben muß.

4) Delpino, Funzione mirmecofila nel regno vegetale I, p. 268.

5) Schimper, A. F. W., Die Wechselbeziehungen zwischen Pflanzen und Ameisen im tropischen Amerika. Jena 1888. 
gehobenen langgestreckten Sattels, der an beiden Enden allmählich abfällt, sodaß das Nektarium auf der Längsansicht trapezoidisch aussieht (Fig. 24, n). Der Scheitel der Drüse wird von einer trogförmigen Längsfurche durchzogen - Delpinos ${ }^{1}$ ) „cratere compresso lineare" —, aus der ich öfter Nektar quellen sah, der nach Delpin $0^{2}$ ) von Ameisen reichlich aufgesucht wird. Das Nektarium ist das größte aller von mir gesehenen und scheint überhaupt eines der größten extrafloralen Nektarien zu sein, da der soeben erwähnte Forscher in seiner zitierten Monographie von ihm sagt: „poche piante hanno nettarii piu elaborati e vistosi“. Ich fand an den im Göttinger botanischen Garten kultivierten Exemplaren Nektarien, deren Sekretionsfurche bis zu $13 \mathrm{~mm}$ lang war; als Durchschnittslänge des sezernierenden Gipfels ergab sich für die daselbst gezogenen Pflanzen $6 \mathrm{~mm}$. Soweit die Drüse reicht, biegen die beiden Flügel, die die Blattspindel kontinuierlich begleiten, bogenförmig nach außen aus. Während die Stipulardornen an den zuletzt entwickelten Blättern nicht mehr kräftig ausgebildet wurden, war die Drüse auch an den Herbstblättern stets gleich üppig wie an den früheren gestaltet.

\section{a) Sezernierendes Nektarium.}

Für die nachfolgende anatomische Darstellung möchte ich den Ausdruck "Nektarium“ dahin präzisieren, daß darunter nur derjenige Teil der ganzen Prominenz verstanden werden soll, welcher drïsigen Charakter zeigt.

Die Gestalt dieses Nektariums ist auf Querschnitten durch den Blattstiel ungefähr flaschenförmig und an besten aus der beigegebenen Figur (Fig. 25) zu ersehen. Sie kann ziemlich variieren und zeigt nicht selten in der Höhe der beiden Flügel auf jeder Seite eine bogenförmige Ausbiegung nach außen hin (Fig. 26, b). Das Nektarium schiebt sich, was von außen nicht zu erkennen ist, an seinen beiden Längsenden noch eine kurze Strecke weit kegelförmig unter das Rindengewebe des Blattstieles (Fig. 27, n). Das Drüsengewebe ist im vorliegenden Fall nicht gerade durch die Kleinheit seiner Elemente ausgezeichnet - es besitzt die größten mir bekannt gewordenen Drüsenzellen -, wohl aber durch zarte Membranen, Plasmareichtum, große Kerne und fast lückenloses Aneinanderschließen der einzelnen Zellen charakterisiert. Im Zentrum des Nektargewebes befinden sich, durch größere Interzellularen abgegrenzt, unregelmäßig gestaltete, neist isodianıtrische, abgerundete Elemente, an einigen Stellen auch sklerenchymatisch verdickte Zellen, deren Wandungen später reich mit Poren versehen sind und mit Phloroglucin und Salzsäure rot, nit Chlorzinkjod gelb werden, also verholzt erscheinen (Fig. 25, $m$ ).

Um dieses zentrale Gewebe herum liegen die eigentlichen Drüsenzellen radiär angeordnet und zwar je nach der Richtung

1) 1. c.

2) ibidem. 
der Stoffwanderung palissadenartig gestreckt, dem Prinzip der Stoffleitung auf kürzestem Wege gemäß. Diese Anordnung tritt am deutlichsten an den äußersten peripheren Schichten (Fig. 25, $и$; Fig. 28, и) des Nektariums hervor.

Dem Scheitel der Drüse fehlt eine Epidermis im physiologischanatomischen Sinne (Fig. 29, S). Die Außenzellen der sezernierenden Medianfurche sind rundlich ${ }^{1}$ ), bedentend dünnwandiger und kleiner als die auf beiden Seiten der Drüsenmündung befindlichen Zellen. Letztere haben sehr stark verdickte und kutinisierte Membranen; gleiches gilt auch noch für drei weitere tiefer gelegene Zellschichten (Fig. 29, l).

Das ganze Nektarium wird ringsum — den Scheitel ausgenommen - von ein bis drei Zellschichten umscheidet, die sich scharf von dem umgebenden Gewebe abgrenzen, in frischen Schnitten grünlich erscheinen und mit Eisenchlorid am schnellsten und am tiefsten schwarz werden. Sehr deutlich treten sie auf nit Hämatoxylin gefärbten Präparaten hervor; während das Drïsengewebe selbst durch die stark gefärbten Membranen bläulich erscheint, bleiben sie vollkommen farblos. Ihre Zellen stoßen dicht aneinander, haben polyedrische Form und zeigen reichliche Granulationen (Chlorophyll) im Plasma. Díe innerste, unmittelbar an die palissadenartig gestreckten Drüsenzellen grenzende Schicht dieser Zelllagen hat ziemlich zarte Wände, die mit Hämatoxylin farblos bleiben, mit Chlorzinkjod gelb und mit Phloroglucin und Salzsäurc blaßrot werden, also "verholzt" sind; und zwar sind sowohl die Radialwände als auch die Tangentialwände verholzt (Fig. 25, s; Fig. 28, s). Bisweilen scheint es, als ob hie und da die Verholzung an den Tangentialwänden schwächer wäre.

Das Nektarium wird durch Gefäßbündel, und zwar besonders Phloenielemente, innerviert; sie endigen mit Zellen, die wohl den "Übergangszellen" A. Fischer's an die Seite zu stellen sind. Daß die scheide gerade an dergleichen Stellen (Fig. 28, s), wo ein inniges Aneinanderschließen der einzelnen Elemente stattfindet, die Reaktion auf ,verholzte" Membranen zeigt, scheint mir ein sicheres Zeichen dafür zu sein, daß sie trotzdem für gelöste Stoffe leicht durchgänglich sein muß, wie denn überhaupt ihr Plasmareichtum schon darauf hindeutet, daß sie nicht nur als mechanischer Teil fungiert, sondern auch eine wesentliche aktive Arbeitsbestimmung hat.

Die eben erwähnten Gefäßbündel entspringen, soweit sie am Fub (Fig. 25, f) der Drüse endigen, wohl nur aus dem Phloemteil der beiden an das Nektarium grenzenden Stränge der Haujtgefäßbündelmasse; die an den beiden Seitenwänden der Drüse endigenden zuleitenden Elemente nehmen ihren Ursprung meist ebenfalls aus den eben genannten Strängen, bisweilen (z. B. in dem Fig 26 gezeichneten Falle) jedoch aus denen der Nebengefäßbündel. Diese Gefäßbündel an den Seitenwänden des Nek-

1) Genau gleich sehen die Sekretionszellen des Nektariums von Acacia neriifolia aus, das eine grubenförmige Vertiefung des Phyllodiums bildet. 
tariuns bestehen aus dreierlei Gewebe: zum größten Teil, und zwar an der Innenseite, aus Phloemelementen, in der Mitte aus spiralig' verdickten Tracheiden und an der Außenseite aus sklerenchymatisch verdickten Elementen. Sie bilden nicht etwa eine kontinuierliche Platte zu jeder Seite der drüsigen Emergenz, sondern in regelmäßigen Abständen gehen an jeder Seite der Prominenz etwa 30 Gefäßbündelstränge empor, die aus der Hauptbündelmasse bezw. den Gefäßbündeln der Flügel entspringen (Fig. 30); sie breiten sich fächerförmig aus und endigen in feiner Verteilung. Durch die so erreichte Flächenvergrößerung ermöglichen sie eine erhöhte und mehr gleichmäßige Stoffleitung.

Das sezernierende Nektarium ist so enorm reich an Gerbstoff, daß es auf mit Kaliumbichromat behandelten Blattstielen schon makroskopisch durch dunkle Färbung scharf hervortritt. Im Bereich des Drüsengewebes lassen sich 3 Gerbstoffzonen unterscheiden: in der Mitte ist der Gerbstoff in Form großer dunkelbrauner Ballen ausgefällt, dann folgen die 2 bis 5 Schichten langgestreckter Palissadenzellen mitheller tingierten, kleineren Gerbstoffkugeln (sehr oft sieht man in diesen Zellen je einen großen Gerbstoffballen an beiden Enden und dazwischen perlschnurartig eine oder zwei Reihen kleinerer Gerbstoffkugeln); in der farblosen Schicht wiederum ist der Gerbstoff so ausgefällt wie im Zentrum. Torstehende Angaben gelten aber nur in großen Zügen; es kommen auch im Zentrum und in den farblosen Schichten öfter kleine Gerbstoffkugeln vor.

Gerbstoff findet sich außerdem in der Epidermis, den hypodermalen Schichten des Rindengewebes, ab und zu auch im Phloem und sehr spärlich im Mark. Die zuleitenden Gefäßbündelelemente an den Seiten des Nektariums sind gerbstofffrei.

Eisenchlorid zeigt die gleiche Verteilung der Gerbstoffe an, wie Kaliumbichromat.

Wurden Schnitte mit Fehling'scher Lösung zum Sieden erhitzt, so fand keine Reduktion zu Kupferoxydul statt, wohl aber fiel der Gerbstoff dunkelbraun aus.

Stärke fehlt inı Drüsengewebe.

\section{b) Entwicklungsgeschichte.}

Das extraflorale Nektarium von Acacia comigera geht nicht allein aus dem Dermatogen hervor, sondern es beteiligen sich zum groben 'Teil die periblematischen Elemente des Rindengewebes an seinem Aufbau; es bildet sich allmählich als Teil einer Emergenz heraus.

Der erste von mir beobachtete Entwicklumgszustand der ganzen Emergenz, aus der sich später das Nektarium herausdifferenziert, ist ein kleiner ellipsoidischer, in der Nitte 3. Schichten dicker \%ellhöcker, der an Grunde zwischen 2 kräftigen nach außen vorgewölbten Gewebeleisten, den späteren Flügeln, liegt. In diesem Stadium hat das sämtliche Gewebe des Blattstieles noch durchaus den ('harakter cines Meristems; in der Hauptbünlelmasse 
sind die Prokambiumstränge gerade angelegt, die der beiden Flügel sind noch nicht angedeutet. Fertig ausgebildet sind dagegen schon jetzt keulenförmige Trichome, die aus der Epidermis der beiden seitlichen Gewebeleisten entspringen und reich an Gerbstoff sind. Diese Haare (cf. Fig. 31, t) stehen besonders zahlreich auf der Innenseite der beiden Flügel und legen sich an der Einbuchtung oft übereinander, indem sie ersichtlich eine Schutzeinrichtung für die junge Emergenz bilden.

Wenn die Prokambien der Flügel erkennbar werden und eben die primären Gefäße im Hauptholzkörper ausgebildet werden, besteht die junge Prominenz in ihrem mittleren Teil aus etwa 12 Zellschichten (Fig. 31, e m). Auf diesem Stadium wird der Fuß (Fig. 25, f, Fig. 31, $f$ ) des späteren Nektariums durch 2 bis 4 Zellschichten von den Prokambiumsträngen der Hauptgefäßbündelmasse getrennt, die Epidermis ist jetzt auch am Scheitel noch deutlich erkennbar, späterhin ist sie weniger abgegrenzt, da sie sich durch Periklinen unregelmäßig teilt (Fig. 31, e p).

Im weiteren Entwicklungsgang bleibt die Emergenz zunächst in ihrem Wachstum merklich hinter dem der beiden Flügel zurück und vergrößert sich bis zur Anlage der primären Gefäße der Flügel nur sehr langsam (Fig. 32, e m). Von da ab beginnt jedoch ein kräftiges akropetales Anwachsen der Emergenz, die sich durch lebhafte Teilungen in radialer Richtung streckt und bald in der Mitte des Scheitels eine kleine Einbuchtung - die spätere nektarausscheidende Furche - erhält dadurch, daß das Wachstum der medianen Zone den Zellen des peripheren Randes gegenüber zurückbleibt. So wächst die Prominenz alsbald bis zur Höhe der beiden Flügel empor.

Während bis dahin die ganze Emergenz aus gleichförmigen meristematischen Zellen bestand, beginnt nun allmählich das Nektarium sich herauszudifferenzieren. Aus dem primären Meristem bilden sich nach und nach zu beiden Seiten des mittleren Teiles, der definitiven Form entsprechend, in der Längsrichtung der Drüse gestreckte Initialstränge aus, die mit der fortschreitenden Gewebedifferenzierung um so deutlicher werden, als ihre Elemente sich wesentlich nur in der Längsrichtung teilen, während die Nachbarzellen durch Querteilungen mehr isodiametrische Form behalten. Aus diesen Prokambien entstehen dann in gewöhnlicher Weise die Gefäßbündelelemente zu beiden Seiten des Nektariums; für den Fuß der Drüse gilt das gleiche.

Durch intensives Wachstum drängt die Emergenz die Flügel schließlich an die Seite; der Fuß des Nektariums bleibt während der ganzen Entwicklung zienılich unverändert, die charakteristische Anordung der Drüsenzellen kommt erst sehr spät zustande.

Der Gerbstoff tritt bereits in dem noch durchaus meristematischen Stadium (Fig. 31, g) auf; er ist zuerst in Form kleiner lichtbrechender Köruchen in den Epidermzellen des späteren Nektariums sichtbar und entsteht hier allem Anschein nach durch chemische Umsetzungen im Plasma. Vom Scheitel aus schreitet die Bildung des Gerbstoffes allmählich gegen die Basis fort, und 
zwar erscheinen zunächst etwa vier mediane Zellreihen - das spätere Drüsenzentrum - mit Gerbstoff angefüllt. Dann beginnt auch, wiederum vom Scheitel anfangend, in dem übrigen Gewebe die Gerbstoffbildung. Bald treten die kleinen Kügelchen zu größeren zusammen, und sobald die Drüse etwa gleiche Höhe nit den Flügeln erreicht hat, sind bereits etwa 3 bis 5 größere Gerbstoffballen in den einzelnen Zellen vorhanden, am größten da, wo der Gerbstoff zuerst auftrat.

Es sei hier bemerkt, daß in frühen Stadien der Gerbstoff durch Alkohol nicht ausgezogen wurde, während dies in sezernierenden Nektarien der Fall war.

\section{Zusammenfassung und Kritik.}

Nachdem im vorhergehenden eine Anzahl extrafloraler Nektarien entwicklungsgeschichtlich-anatomisch getrennt behandelt worden sind, will ich nunmehr versuchen, die bis jetzt gefundenen Ergebnisse zusammenzufassen. Dabei werde ich ab und zu auch einige andere im vorigen ausfïhrenden Teil nicht erwähnte extraflorale Nektarien, deren Bearbeitung noch nicht abgeschlossen ist, mit in Betracht ziehen.

\section{Verbreitung der extrafloralen Nektarien; Drüsengewebe, sezernierende Epidermis, Gefässbündel.}

Zunächst bestätigten auch die von mir untersuchten Objekte, daß bei extrafloralen Nektarien in vielen Punkten eine große Mannigfaltigkeit, dann aber wiederum manches gemeinsame zu konstatieren ist.

Einerseits kann bei verschiedenen Familien dieselbe Drüsenform wiederkehren, z. B. bei Polygonaceen und Malvaceen, bei Verbenaceen (Clerodendron) und Bignoniaceen; anderer'seits können in derselben Familie verschieden gestaltete Nektarien auftreten z. B. bei den Leguminosen; ja sogar in derselben Gattung können die Nektarien entweder beträchtliche Abweichungen zwischen den einzelnen Spezies zeigen z.B. bei Acacia, Passiflora, oder manchen Arten ganz fehlen, z. B. Vicia. In morphologisch-entwicklungsgeschichtlicher Hinsicht sind die extrafloralen Nektarien sehr ungleich, entweder reine Dermatogenprodukte (z. B. Polygonaceen) oder aus dem Periblem genetisch herzuleiten (z. B. Acacia cornigera, Ricinus, Prunus $\left.{ }^{1}\right)$, Sambucus).

W. J. Behrens') gibt an, daß die Nektarien der Blüten immer aus mehreren Teilen bestehen: „der eine, wichtigste, findet sich bei allen Nektarien und ist im Vorhergehenden mit dem Namen Nektariumgewebe bezeichnet worden." Für extraflorale Nektarien gilt dieser Satz nicht, da zum Beispiel den Polygonaceen ein Drüsengewebe fehlt.

1) Reinke, l. c. p. 127.

2) 1. c. p. 371 . 
Die Angabe Morinisi): „L'estensione del parenchima è in rapporto inverso col grado di sviluppo e di differenziazione dell' epidermide" ist unrichtig, wie zum Beispiel ein Vergleich zwischen Muehlenbeckia, Drynaria und Lignstrum zeigt. Es scheinen sich nach unseren jetzigen Kenntnissen keine Regeln für eine derartige Beziehung aufstellen zu lassen.

Die im speziellen Teil dieser Arbeit behandelten Fälle waren in einer kontinuierlich fortlaufenden Reihe angeordnet, der Ausbildung eines Drüsengewebes entsprechend. Sie begann mit Nektarien, die nur aus einer Gruppe besonders modifizierter Epidermzellen (Drüsenhare) bestehen, also typische Hautdrüsen sind: Polygonaceen.

Darauf folgten Nektarien, deren sezernierender Teil gleichfalls Trichome sind, die, sämtlich nach einem Prinzip gebaut, bei den verschiedenen Arten in Einzelheiten variieren.

Bei Gossypinm ist ein subglandulares Gewebe vorhanden, das schon etwas von dem umgebenden Parenchym abweicht, bei Tecoma ist ein derartiges Gewebe noch schärfer ausgeprägt und gehört physiologisch sicher zum Nektarium. Bei Ligustrum dagegen besteht das Nektarium nicht nur aus ,pili glandulosi“", sondern es besitzt darunter außerdem ein wohl differenziertes Drüsengewebe, das allmählich in das umgebende Mesophyll übergeht.

Die beiden anderen Objekte, Polypodiaceen und Acacia cormigera haben Nektarien. die einen gut ausgebildeten Drüsenkörper besitzen, der bei den Farnen allmählich in das benachbarte Mesophyll übergeht, bei Acacia dagegen scharf begrenzt ist. Ein besonderer sezernierender Teil fehlt in diesen zwei Fällen.

Sehr häufig besteht die sezernierende Oberfläche der extrafloralen Nektarien aus Pal issadenzellen oder doch aus prismatisch gestreckten Zellen; ich beobachtete dergleichen außer den im speziellen Teil angefïhrten Fällen bei Cassia arborescens Vill., $C$. marylandica Linn., Clerodendron fragrans TVilld., Cl. ixoriflorum Hassk., Hippomane Mancinella Linn., Hura crepitans Linn., Modecca lobata Jacq., Momordica cochinchinensis Spreng., Passiflora coerulea Linn., Passiflora edulis Sims., Populusalba Linn.. P. candicans Ait. Hort. Kew, P. monilifera Ait. Hort. Kew, P. tremula Linn., Prumus Armeniaca Linn., P. arium Linn.. P. Laurocerasus Linn., P. Padus Linn., Turnera angustifolia Mill. Daß solche Zellform für die vorliegende Funktion zweckmäßig ist, indem hierdurch die Oberfläche vergrößert wird, leuchtet ohne weiteres ein. WTir haben hier wieder ein Beispiel dafür, daß der Bau eines Gewebes seiner Leistung entspricht.

Stadler ${ }^{2}$ ) fand für die Nektarien der Blüten, „daß die Vasalien einen integrierenden, nie fehlenden Bestandteil. wenn auch nicht des Nektariumgewebes, so doch des Nektariumbodens bilden". Nach meinen Beobachtungen werden auch extraflorale Nektarien

1) 1. c. p. 369 .

2) Stadler, Beitrïge zur Kenntnis der Nektarien und Biologie der Blüten. Diss. Zürich 1886 . p. 69. 
sehr oft von Gefäßbündelendigungen innerviert, und nicht selten (z. B. Acacia cornigera, Tecoma radicans) machen die Gefäßbündel im Bereich der Drüse Abweichungen vom normalen Verlauf, wie es Stadler auch für florale Nektarien öfter angibt. Bei Polygonaceen und Gossypien jedoch findet keine besondere Innervation der Nektarien statt; man kann hier vielleicht die Lage derselben im Rindengewebe, das ja auch Kohlehydrate leitet, für das Fehlen der Gefäßbündel geltend machen.

\section{Lageverhältnisse, meristematischer Charakter des Nektariumgewebes.}

Die Entwicklungsgeschichte zeigte, daß Lage und Gestalt der Drüse in jugendlichen Stadien bisweilen nicht unerheblich von den definitiven Verhältnissen abweichen.

Ein durch aktives Wachstum zustande gekommenes Einsenken des Drüsenkörpers, wie es Corrensi ${ }^{1}$ ) für Dioscorea und Zimmermann ${ }^{2}$ ) für Fagraea angeben, habe ich bei den von mir entwicklungsgeschichtlich untersuchten Nektarien nicht beobachtet; wenn die ausgebildete Drüse unter dem Niveau der umgebenden Epidermis liegt, so wies vielmehr die Entwicklungsgeschichte nach, daß die definitive Lage durch Überwallung seitens des Nachbargewebes zustande kommt ${ }^{3}$ ).

Bei den betreffenden Objekten wächst in den ersten Stadien die jungeDrüse stets zunächst aktiv eine Strecke über das umgebende Niveau empor, vollendet dann meist im großen und ganzen ihren Aufbau, bis das sie tragende Organ sich zu strecken beginnt. Von da ab verhalten sich die Drüsen passiv, sie bleiben auf dem embryonalen Zustand verharren, ohne an der Vermehrung und Vergrößerung der Nachbarzellen teilzunehmen. Auf solche Weise kann dann bei dem späteren Längen- und Dickenwachstum des betreffenden vegetativen Teiles durch das inaktive Verhalten des zur Drüse gehörenden Zellkomplexes einmal eine Erhebung des Nachbargewebes und dann eine konzentrisch auf die Drüse zu gerichtete Streckung der Zellen desselben erfolgen. Auf derartige Wachstumsvorgänge sind mechanisch zurückzuführen z. B. die eingesenkte Lage der Nektarien bei Gossypium und Polygonaceen, die Streckung des Nachbargewebes bei Tecoma. Auch die strahlenförmige Gruppierung der Epidermzellen bei den Farnnektarien ist so durch ein Nichtbeteiligen des Nektariums am Flächenwachstum der Lamina erklärbar.

Man kann demnach manche extraflorale Nektarien, wie z. B. die der Polypodiaceen, als Hemmungsbildungen in gewissem Sinne bezeichnen, indem an diesen Stellen des Blattes das Gewebe

1) 1. c. p. 669 .

2) Zimmermann, Über extranuptiale Nektarien einiger Fagraea-Arten. Ann. d. j. bot. de Buitenzorg. Bd. 18. 1902. p. 5.

3) cf. Ewart, On the Leaf-glands of Ipomoea paniculata in Annals of Botany. Vol. 9. 1895. p. 280. 
auf dem embryonalen Zustand verharrt (gerade Epidermiswände, unausgebildete Spaltöffnungen) und meristematisch bleibt, nur mit dem Unterschied, daß es keine Teilungen mehr eingeht.

Für das Drüsengewebe von Ligustrum gilt ein gleiches. Denn wie die Entwicklung lehrt, ist auch hier zunächst kein anatomischer Unterschied zwischen dem späteren Nektariumgewebe und dem noch meristematischen späteren Mesophyll zu konstatieren, selbst dann noch nicht, wenn die Epidermis bereits zu Trichomen umgestaltet wird; bei der Entfaltung des jungen Blattes bleibt das Gewebe über den Drüsenhaaren so, wie bisher das übrige Mesophyll war, nämlich kleinzellig und ohne nennenswerte Interzellularen.

Ein intensives aktives Wachstum hingegen besitzt das Nektarium von Acacia cornigera, das, an der Basis im großen und ganzen ziemlich unverändert bleibend, sich allmählich immer mehr hervorschiebt und schließlich die Flügel des Blattstieles an die Seite drängt.

\section{Verdickter und ,verkorkter" Stielteil der nektar- ausscheidenden Trichome.}

Bei den von mir untersuchten Nektarien, die mittels Drüsenhaaren sezernieren, lassen sich stets drei Teile an dem einzelnen Trichom unterscheiden: der Fuß, der Stiel und das sezernierende Köpfchen. Eine vergleichende Betrachtung zeigt, daß, wenn auch Gestalt und Ausdehnung der Trichome bei den verschiedenen Familien, Gattungen und Arten erheblich differieren, doch bei allen dasselbe Bauprinzip wiederkehrt, besonders auffallend in der Beschaffenheit des Stieles. Bei sämtlichen von mir untersuchten derartigen Nektarien sind die Radialmembranen des Stieles verdickt nnd bleiben auf mit Hämatoxylin gefärbten Schnitten farblos. Dasselbe ist bei Vicia amphicarpa Dorthes, V. Faba Linn. und V. sepium Linn. der Fall. Der Inhalt der Stielzelle zeigt meist reichliche Granulationen.

Die Entwicklungsgeschichte belehrte mich, daß dergleichen in jungen Stadien nicht zu konstatieren ist und diese Umgestaltung und Veränderung erst kurz vor Beginn der Sekretion eintritt, nachdem in dem Zellkomplex der Drüse die abschließenden Teilungen geschehen sind. Bei Tecoma scheint dafür keine so strenge Gesetzmäßigkeit zu herrschen, da ich hier öfter beobachtete, daß schon in bedeutend früheren Stadien die Stielzellen nach Inhalt und Membran von ihrer Ungebung abweichen. Diese chemische und physikalische Veränderung der Stielmembran tritt zunächst in den Radialwänden auf, ¿die mit Chlorzinkjod jetzt eine gelbbraune Färbung annehmen; man dürfte sie demnach als ,.verkorkt" bezeichnen, wenn man diesen Ausdruck, der ja bekanntlich bis jetzt immer noch nur ein Sammelname für verschieden metamorphosierte Zellmembranen ${ }^{1}$ ) ist, überhaupt anwenden will.

1) cf. Czapek, Biochemie I, p. 572. 
Was die Aufgabe dieser so auffallend gefestigten Zelle bezw. Zellschicht anlangt, so könnte man einmal vermuten, daß sie, wie es Haberlandt ${ }^{1}$ ) für die Stielzelle der Hydathoden annimmt, "gewissermaßen den mechanischen Apparat des ganzen Organs repräsentiert, indem ihre oft stark verdickten und fast immer ausgiebig cutinisierten Seitenwände einen festen Ring bilden, der die Aus- und Eintrittsöffnung für das Wasser stets gleich weit erhält." Dann dürfte man ihr vielleicht auch eine schützende Rolle gegen die Druckspannungen, die während der Sekretion im Drüsenhaar herrschen müssen, zuschreiben. Schließlich scheint bisweilen auch von den dem Fuß zu gelegenen Stellen aus die Verkorkung auf die Tangentialwände der Basalzellen überzugreifen. Ich sah wenigstens öfter bei Muehlenbeckia sagittifolia, Polygonum dumetorum und Polygonum multiflorum, daß nach Sistierung der Sekretion die Fußzellen, manchmal auch die darunter liegenden subepidermalen Zellen, verdickte und verkorkte Membranen besaßen. Die Pflanze scheint also in manchen Fällen danach zu trachten, nach beendigter Sekretion einen möglichst guten Abschluß nach auBen zu erzielen, was ja gerade bei solch labilen Gebilden, wie es die Drüsentrichome sind, leicht verständlich ist. Denn da der Nektar ungeschützt auf der freien Oberfläche liegt, so bildet er ein günstiges Substrat für Pilzsporen, die ich denn auch nicht selten auf Nektarien gekeimt fand.

Solch eine verkorkte Schicht beobachtete ich außer bei sämtlichen im speziellen Teil beschriebenen Trichomen noch bei den extrafloralen Nektarien von Dioscorea alata, Clerodendron fragrans und $\mathrm{Cl}$. ixoriflorum. Aus der einschlägigen Literatur entnehme ich, daß gleiches auch für die Nektarien von Nepenthes und Ipomoea bekannt ist ${ }^{2}$.

Welche Bedeutung dieser Stielzelle für die Stoffumsetzung zukommt, läßt sich bei dem heutigen Stande der Mikrochemie nicht entscheiden.

\section{4. „Verholzte“ Scheide.}

Eine nach einem anderen Typus gebaute "Schutzscheide“ ist die bei Acacia cornigera geschilderte Schicht, deren Wände mit Hämatoxylin farblos bleiben. In diesem Fall liegt aber eine den ganzen Drüsenkomplex gleichmäßig und lückenlos umschließende Scheide vor, deren zarte Membranen, obwohl sie die Reaktion auf sogenannte "verholzte“ Zellwände geben, für Inhaltstoffe leicht durchlässig sein müssen. Eine sich gleich verhaltende Scheide, deren Membranen mit Phloroglucin und Salzsäure auch auf der Tangentialseite rot werden, fand ich ferner bei den extrafloralen Nektarien von Momordica cochinchinensis Spreng., Luffa cylindrica II. Roem., Acacia Lichtensteinii, A. longifolia Willd., A. longifolia

1) Haberlandt, Anatomisch-physiologische Untersuchungen über das tropische Laubblatt I. Sitzb. Wien. Akad. Bd. 103. Wien 1894. p. 535.

${ }^{2}$ ) Correns, l. c. p. 659, gibt eine verkorkte Schutzscheide un das Nektarium von Dioscorea sativa und D. Batatas an. 
v. Sophorae, A. neriifolia A. Cunn., A. saligna Wendl. Sie scheint demnach bei den extrafloralen Nektarien der mit Phyllodien versehenen Akazien sehr verbreitet zu sein.

Eine befriedigende Erklärung für diese Erscheinung habe ich noch nicht gefunden; es mag sein, daß die Aufgabe dieser Scheide die einer Druckgrenze im Sinne von de Vries ${ }^{1}$ ) ist, da höchst wahrscheinlich während der Sekretion durch das gestörte osmotische Gleichgewicht zeitweise große Druckdifferenzen zwischen dem. Drüsengewebe und dem Nachbargewebe herrschen; aus dem Inhalt (dichtes Plasma, Chloroplasten) dieser Zellschicht erhellt, daß ihr wohl auch für die Stoffleitung eine wichtige Funktion zukommt.

Ich möchte annehmen, daß man dergleichen mechanische Scheiden noch in manchen anderen Fällen finden wird, sobald man extraflorale Nektarien fernerhin von solchen Gesichtspunkten aus bearbeitet.

\section{Inhalt, Gerbstoff.}

Was die Inhaltstoffe der bearbeiteten Nektarien anlangt, so habe ich ihnen keine besondere Beachtung zugewendet, da eine diesbezügliche Untersuchung nicht im Plan der vorliegenden Arbeit lag. Es existieren ja auch einerseits schon mancherlei Angaben darüber, z. B. in den erwähnten Arbeiten von Bonnier und Morini, und andererseits ist es bei dem jetzigen Stande der Mikrochemie oft unmöglich, auch nur annähernd alle Inhaltstoffe genau festzustellen, da es meist an zuverlässigen Reagentien fehlt.

Um sicher zu sein, daß die untersuchten Drüsen Nektarien waren, genügte es für mich, in der abgesonderten Flüssigkeit eine Zuckerart nachzuweisen. Die Reaktion mit Fehling'scher Lösung gelang außer bei sämtlichen im speziellen Teil näher geschilderten Spezies bei den extrafloralen Nektarien von Acacia Lichtensteinii, A. lophantha Willd., A. cyanophylla Lal., A. Melanoxylon R. Br., A. neriifolia A. Cunn., A. saligna Wendl., Actinostemma paniculatum Maxim., Cassia marylandica Linn., Clerodendron fragrans Willd., Cl. ixoriflorum Hassk., Cyclanthera explodens Naud., Modecca lobata Jacq., Momordica cochinchinensis Spreng., Neptumia oleracea Lour., Passiflora adenopoda D. C., P. coerulea Linn., $P$. gracilis Jacq., Pithecolobium dulce Benth., Populus candicans Ait. Hort. Kew., P. monilifera Ait. Hort. Kew., Prums Armeniaca Linn., $P$. avium Linn., P. Padus Linn., P. persica Stokes, Sambucus nigra Linn., S. racemosa Linn., Syringa chinensis Willd., S. persica Linn., Vicia amphicarpa Dorthes, V. Faba Linn., V. sepium Linn.

Da mir bei Beginn der Arbeit in einigen Nektarien ein reicher Gerbstoffgehalt auffiel, so wandte ich meist - nicht immer diesen „gerbstoffartigen Körpern" einige Beachtung zu.

Wie bereits friher angegeben wurde, sind die extrafloralen Nektarien oft so enorm reich an Gerbstoff, daß dieser schon auf den ersten Blick die Drïse erkennen läßt,

1) Zitiert nach Haberlandt, Physiologische Pflanzenanatomie III. Aufl. p. 328 . 
wenn man ihn irgendwie ausfällt. Teilweise ist das Drüsengewebe so vollgepfropft mit Gerbstoffen, daß man nicht umhin kann, diesen Körpern, die die sogenannten "Gerbstoffreaktionen" geben, irgend eine Beziehung zu dem gebildeten Zucker zuzuschreiben.

Wie aber die Bildung des Zuckers mit dem auftretenden Gerbstoff zusammenhängt, darüber läßt sich bei dem heutigen Stand der Gerbstoffphysiologie ${ }^{1}$ ) nichts sicheres sagen; ebenso sind auch alle anatomisch-physiologischen Spekulationen über die Rolle des bereits im Meristem auftretenden Gerbstoffes zur Zeit noch verfrüht.

Gerbstoff wurde bis jetzt in Nektarien beobachtet von Stadler ${ }^{2}$, der für die Nektarion der Blüten angibt, „daß in selteneren Fällen zuckerhaltige Sekrete auch aus Gerbstoff hervorgehen können."

Behrens ${ }^{3}$ ) fand nicht selten Gerbstoffe, schreibt ihnen aber keine besondere Bedeutung zu; gleiches gilt für Morini $\left.{ }^{4}\right)$, der besonderes Gewicht auf die Inhaltstoffe legte, diese Gerbstoffe jedoch in einigen Fällen ïbersehen hat.

Rathay ${ }^{5}$ ) fand Gerbstoff in den ncktarabsondernden Trichomen einiger Melampyrum-Arten. Haberland $t^{6}$ ) gibt Gerbstoffballen in den Drüsenzellen von Vicia sepium an.

Aufrecht7) schließt sich der Ansicht von Krauss an, „daß ein Eintritt des Gerbstoffes in den Stoffwechsel als völlig ausgeschlossen gelten muß", und hält ihn dort, wo er in extrafloralen Nektarien auftritt, für eine Schutzvorrichtung im Sinne Stahls.

Calciumoxalat, dem Aufrecht8) die von Kohl aufgestellte "Rolle des Transporteurs für die Kohlehydrate" zuschreibt, fand ich unter anderem in größerer Menge in den extrafloralen Nektarien von Hura crepitans Linn., Passiflora coerulea Linn., P. edulis Sims., Populus alba Linn., P. tremula Linn.. Prumus avium Linn., Sambucus nigra Linn., Turnera angustifolia Mill.

\section{Vorkommen homologer Gebilde.}

Wie bereits betont, haben die Drüsenhaare der Nektarien Homologa in den an topographisch gleichen Stcllen vorkommenden Einzeltrichomen, die meist die Zellteilungen schon vollendet haben, wenn das Nektarium sich erst anzulegen beginnt.

1) Czapek, Biochemie II, p. 588; Moeller, Anatomische Untersuchungen über das Vorkommen der Gerbsäure. Berichte d. deutsch. bot. Gesellsch. Bd. 6 . 1888, p. IXXX; Berthold, Untersuchungen zur Physiologie der pflanzlichen Organisation I. 1898, p. 13; II 1904, p. 19.

2) 1. c. p. 72 .

3) l. c. p. 446 .

4) l. c. p. 375 .

5) 1. c. p. 60 .

6) Haberlandt, Das tropische Laubblatt II. Sitzb. d. Wien. Akad. 1895. Bd. 104. p. 101.

7) 1. c. p. 42 .

8) l. c. p. 43 . 
Haberlandt ${ }^{1}$ ) fand, daß bei Vicia sepium „die Keulenhaare der extranuptialen Nektarien genau denselben Bau besitzen, wie die als Wasserdrüsen fungierenden Keulenhaare auf den Oberseiten der jungen Fiederblättchen". Er schließt²): „In phylogenetischer Hinsicht dürften die meisten Nektarien, gleich den Digestionsdrüsen der Insektivoren, von Hydathoden abzuleiten sein, und zwar sowohl von Epithem- wie von Trichomhydathoden." Haberlandt nimmt also einen Funktionswechsel dieser Hydathoden an.

Ich untersuchte in den behandelten Fällen die Einzeltrichome nach Inhalt und Funktion nicht näher; anatomisch ähneln einige (z. B. die von Tecoma und Ligustrum) täuschend manchen von Haberlandt ${ }^{3}$ ) abgebildeten Hydathoden, und auch die Verdickung und Verkorkung der Radialmembran des Stieles, die sowohl bei den Einzeltrichomen, als bei den nektarabsondernden Drüsenhaaren

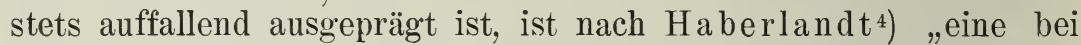
Trichomhydathoden so häufig zu beobachtende Erscheinung".

Es liegt bei der heutigen Wertschätzung der Entwicklungsgeschichte ja recht nahe, auch für die von mir bearbeiteten Nektarien mit Drüsenhaaren an eine phylogenetische Abstammung der in Gruppen stehenden Trichome aus den zerstreut vorkommenden $\mathrm{zu}$ denken. Doch ist immerhin gerade bei Trichombildungen in dergleichen phylogenetischen Betrachtungen einige Vorsicht geboten, und ich glaube, daß zum Beispiel Morinis) zu weit geht, wenn er nur aufgrund der Ähnlichkeit der extrafloralen Nektarien von Clerodendron, Bignonia und Tecoma auf eine phylogenetische Verwandtschaft der Verbenaceen und Bignoniaceen schließen will; herrschen doch nach de Bar $\mathrm{y}^{6}$ ) gerade in Bezug auf Haarbildungen bei den verschiedenen Familien einerseits große Übereinstimmungen, andererseits in natürlichen Familien und selbst Gattungen die mannigfachsten Verschiedenheiten.

Als sicher feststehend kann für die von mir behandelten Spezies mit derartigen extrafloralen Nektarien nur gelten, daß an demselben vegetativen Teil, ja an den entsprechenden Stellen, zweierlei morphologisch gleichwertige, zumteil bis auf strukturelle Feinheiten gleichgebaute (Ligustrum) Drüsenhare vorkommen, und daß die Einzeltrichome das primär Vorhandene sind.

$\mathrm{Ob}$ sich beiderlei Drüsenhaare aus einer Urform nach verschiedenen Richtungen weiter entwickelt haben, oder ob die Nektarien nur eine lokale Anhäufung der zerstreut vorkommenden Trichome sind, ferner, ob beide jemals Analoga waren und im

1) Haberlandt, Das tropische Laubblatt II. Sitzb. d. Wien. Akad. Bd. 104.1895 . p. $100 \mathrm{ff}$.

2) Derselbe, Physiol. Pflanzenanatomie, p. 449.

3) Derselbe, Das tropische Laubblatt Sitzber. d. Wien. Akad. Bd.

103. 1894. Bd. 104.1895.

4) Derselbe, Das tropische Laubblatt II. Sitzb. Wien. Akad. Bd. 104. 1895. p. 97.

5) Morini, l. c. p. 355.

6) de Bary, Vergleichende Anatomie, p. 59. 
Laufe der Phylogenie ein Funktionswechsel eintrat ${ }^{1}$ ), oder ob gar späterhin die Nektarien die Aufgabe dieser Einzeltrichome, die zum größten Teil nach Ausbildung der Nektarien abgestoßen werden, in etwas modifizierter Weise eventuell übernehmen können, darüber sind vorläufig nur Vermutungen anzustellen.

Außer in den zitierten Arbeiten von Morini und Haberlandt finden sich Angaben über dergleichen Homologa noch bei Reinke2), der festgestellt hat, daß die Nektarien am Petiolus von Prunusarten den Blattzahndrüsen morphologisch gleichwertig sind, und bei Rathay ${ }^{3}$ ), (ler die den Drüsenhaaren von Melampyrum arvense homologen kleinen Schüppchen für rudimentäre Drüsenschuppen hält, die „auf einer niederen Entwicklungsstufe stehen blieben."

\section{Bedeutung der extrafloralen Nektarien für die Pflanzen.}

Diese Frage ist von den Autoren, soweit sie nicht von vornherein auf eine Erklärung verzichteten, mannigfach, oft sehr divergierend ${ }^{4}$ ) beantwortet worden.

Ich will von den vielen, oft sehr merkwürdigen Erklärung’en hier nur einige anführen.

Belt und Delpino halten die extrafloralen Nektarien für Anpassungen an Ameisen, die als Gegendienst den Pflanzen einen Schutz gegen Feinde gewähren sollen.

Kerner ${ }^{5}$ ) erblickt in ihnen Schutzeinrichtungen, die den Zweck haben, Ameisen von dem Besuch der Blüten fernzuhalten.

$\mathrm{Sch}$ im per ${ }^{6}$ ), der in sehr verdienstvoller Weise zum erstenmal die Fragestellung präzisierte, kommt nach kritischen Untersuchungen zu dem Schluß, daß in der großen Nehrzahl der Fälle in der amerikanischen Flora die extrafloralen Nektarien Lockmittel für Ameisen darstellen.

Im allgemeinen kann man wohl sagen, daß heutzutage die meisten Forscher sich der Belt-Delpino'schen Auffassung angeschlossen haben und die extrafloralen Nektarien für spezielle Anpassungen an Schutzameisen halten.

Seit jeher fehlte es jedoch nicht an Gegnern solcher Deutung, die den extrafloralen Nektarien nur eine physiologische Aufgabe im Stoffwechsel zuschreiben. So betrachtet Bonnier ${ }^{7}$ ) die extra-

1) Haberlandt, Das tropische Laubblatt II. Sitzb. Wien. Akad. Bd. $104,1895$. p. 102.

2) 1. c. p. 122

3) l. c. p. 67 .

4) Man vergleiche die Angaben Kerners (Pflanzenleben I, p. 220 1888) und Lundströms (Pflanzenbiol. Studien, zitiert nach Just 1887, p. 441) über die extrafloralen Nektarien von Populus tremula.

$\left.{ }^{5}\right)$ Kerner, Die Schutzmittel der Blüten gegen unberufene Gäste p. 56.

${ }^{6}$ ) Schimper, Die Wechselbeziehungen zwischen Pflanzen und Ameisen im tropischen Amerika. 1888, p. 78.

$\left.{ }^{7}\right)$ l. c. p. 206. 
floralen Nektarien als Reservestoffbehälter, und auch Rathay ${ }^{1}$ ) hält die Mehrzahl der extrafloralen Nektarien für Organe, „die in erster Linie bei dem Ernährungsprozesse eine Rolle spielen."

Außerdem wird in manchen kleineren Arbeiten, auf die ich hier nicht näher eingehen kann, die Aufgabe der extrafloralen Nektarien als Attraktion für Ameisen in Frage gestellt.

Ich glaube, und andere haben bereits ähnliches gesagt, daß es zum mindesten voreilig ist, von vornherein alle Pflanzen, die extraflorale Nektarien besitzen, für myrmekophil zu erklären. Es muß doch zunächst festgestellt werden, ob die extrafloralen Nektarien einer Pflanze überhaupt von Ameisen besucht werden - nach $\mathrm{Huth}^{2}$ ) ist dies bis jetzt nur für eine geringe Anzahl von Fällen direkt beobachtet ${ }^{3}$ ) - , und dann ist zu prüfen, ob diese Ameisen der betreffenden Pflanze einen wirksamen Schutz gegen irgendwelche Feinde gewähren

Ist beides bewiesen, so dürfen wir zwar von einem symbiotischen Verhältnis zwischen Pflanzen und Ameisen reden, haben aber immer noch keine Berechtigung, die extrafloralen Nektarien bei allen Pflanzen als nur für die Ameisen entstanden anzusehen, sodaß sie allein zu deren Anlockung dienten.

Ehe wir uns mit einer solchen ausschließlich äußere Faktoren inbetracht ziehenden Erklärungsweise, die im Grunde doch wenig befriedigend ist, begnügen, müssen wir zunächst versuchen, ob wir die Ausbildung und Bedeutung dieser Organe als durch physiologische Vorgänge bedingt zu erfassen vermögen. Dann kommt erst in zweiter Linie die biologische Frage nach der eventuellen Anpassung an Ameisen inbetracht.

Ich bin im Gegensatz zu anderen der Meinung, daß die Beziehung, die heutzutage manche extraflorale Nektarien zu den Ameisen zeigen, in der Hauptsache nur eine Sekundärers cheinung, eine indirekte Anpassung ist, daß ferner nicht alle sogenannten extrafloralen Nektarien ein und demselben Zweck dienen, sondern ungleiche Bedeutung haben.

Pfeffer ${ }^{4}$ ) trennte bereits 1877 in seinen „osmotischen Untersuchungen" die IVasserausscheidung beim Bluten von der in Nektarien, und 1878 gibt auch Bonnier ${ }^{5}$ ) die Beteiligung des Zuckers als osmotisch wirksame Substanz bei der Nektarabsonderung an; später ist durch Pfeffer ${ }^{6}$ ) und seine Schüler ${ }^{7}$ ) dann nachgewiesen, daß die Sekretionsmechanik, sobald einmal der Zucker außen gegeben ist, durch rein osmotische Wirkung eine zeitlang fortdauert; bisweilen kann auch nach $\mathrm{Haupt}^{8}$ ) eine aktive Wasserauspressung

\footnotetext{
1) Rathay, Über extraflorale Nektarien. Verh. d. zool.-bot. Gesellsch. Wien Bd. 39. 1889 p. 20.

2) Zitiert nach Schimper, l. c. p. 63.

3) Nach meinen Beobachtungen werden in unserer Flora von Ameisen rege besucht die extrafloralen Nektarien von: Vicia Faba, Vicia sepinm, Prumus avium und Pteris aquilina.

4) Pfeffer, Pflanzenphysiologie I. 1897. p. 266.

5) 1. c. p. 181 .

6) 1. c. p. $263 \mathrm{ff}$.

7) Wilson, On the cause of the excretion of water on the surface of nectaries. Diss. Leipzig 1881. Haupt, Zur Sekretionsmechanik der extrafloralen Nektarien. Flora 1902.

${ }^{8)}$ l. c. p. 11.
} 
stattfinden, so daß wir es hier mit Übergängen zu Hydathoden zu tun haben.

Also die osmotisch wirksame Substanz ${ }^{1}$, die anscheinend stets das Primäre ist, ist bei vielen Nektarien die äußere Ursache der darauf eintretenden Wassersekretion.

Könnte sie nun nicht vielleicht in manchen Fällen nur das Mittel zum Zweck sein, indem nämlich der ausgeschiedene Zucker durch seine osmotische Wirkung den Wassergehalt des jugendlichen Organs so lange reguliert, bis die jungen Spaltöffnungen dieser Aufgabe selber gewachsen sind? Wäre also vielleicht die bis jetzt für eine Begleiterscheinung gehaltene Wasserausscheidung: nicht ursprünglich gar der Endzweck?

Der absolute Zuckergehalt des Nektariums bleibt nach Bonnier ${ }^{2}$ ) derselbe, nur durch größeres oder geringeres Verdunsten des Wassers variiert die Konzentration des Nektars.

Die Sekretion selbst ist von ausreichender Feuchtigkeit der Luft abhängig und geht dem jeweiligen Grade der Transpiration paralle $l^{3}$.

$\mathrm{Haupt}^{4}$ ) sagt: „Am stärksten fand ich die Absonderung fast allgemein zur Zeit nach der Laubentfaltung, sowie kurz vor Beginn, und bei einigen Pflanzen während der Blüteperiode. In dieser Zeit, wo das kräftigste Wachstum in der Pflanze stattfindet, zeigt sich die innere Disposition zur Nektarabsonderung am stärksten. ${ }^{\prime \prime}$

Nach Bonnier ${ }^{5}$ ) und $\mathrm{Haupt}^{6}$ ) wird der Zucker nach Sistierung der Sekretion meist wieder nach innen resorbiert.

Alles dieses stützt die soeben ausgesprochene Vermutung in nicht geringem Grade.

Zum Schaden der Pflanze kann das Gleichgewicht im Stoffwechsel gestört werden, einmal in dem Stadium der Entfaltung des Blattes - wir sahen, daß oft zu dieser Zeit die Einzeltrichome abgestoßen werden -, wo die kräftigste Stoffzufuhr stattfindet und die jungen Spaltöffnungen die Abgabe des nötigen Wassers in Dampfform noch nicht leisten können (Polypodiaceen), oder ein andermal, wenn der Feuchtigkeitsgehalt der Luft zи groß wird (künstliche Hervorrufung der Sekretion). Für beide Fälle mögen dann manche extrafloralen Nektarien regulatorisch wirken, indem sie einen kontinuierlichen Wasserstrom ermöglichen.

So mag eine derartige Selbstregulierung des betreffenden Organes, die mit dem Erwachsen desselben, überflüssig geworden, aufhört, die primäre Bedeutung mancher - vor einer Verallgemeinerung müssen wir uns hüten - extrafloraler Nektarien sein; sekundär kann dann nicht selten infolge des ausgeschiedenen

1) Nach Bonnier, l. c. p. 85, können außer Saccharose und Glykose auch Dextrin, Gummi, Mannit, $\mathrm{N}$ - oder P-haltige Produkte im Nektar vorkommen.

${ }^{2}$ 1. c. p. 157, 187; cf. auch $\mathrm{Haupt}$, 1. c. p. 9.

3) Morini, l. c. p. 380; Bonnier, l. c. p. 157.

4) l. c. p. 5 .

5) l. c. p. 199.

6) l. c. p. 5 . 
Nektars der Pflanze im Laufe der Zeit ein nicht unerheblicher Vorteil dadurch erwachsen sein, daß die angelockten Ameisen allmählich zu einer Schutzgarde gegen verschiedene Feinde wurden; sie trugen bisweilen wohl auch wesentlich dazu bei, daß die Nektarien sich weiter entwickelten, Saftmale u. s. w. ausbildeten.

Viele extraflorale Nektarien mögen auch heutzutage noch nur die soeben erwähnte spezifische Bedeutung für den Gesamtstoffwechsel des betreffenden Organs besitzen, andere (Polygonum baldschuanicum, Viburnum Opulus) scheinen in Rückbildung begriffen zu sein, doch wären darüber noch eingehende Untersuchungen erforderlich.

Un es noch einmal kurz zu sag'en, möchte ich also für einen Teil der extrafloralen Nektarien annehmen, daß sie primär die physiologische Aufgabe besessen haben bezw. noch besitzen, als Regulatoren für den Wassergehalt des sie tragenden Organes zu fungieren, sei es in jungen Stadien zur Zeit der Entfaltung, sei es, wenn sonst die Transpiration herabgesetzt ist; im Laufe der Phylogenie mag dann allerdings bei manchen die sekundäre Beziehung zu Ameisen in den Vordergrund getreten sein.

\section{Einige Ergebnisse.}

Die extrafloralen Nektarien der Polygonaceen sind reine Epidermalgebilde, ein typisches Drüsengewebe fehlt ihnen. Ein physiologisch zum Nektarium gehörendes, anatomisch nicht scharf ausgeprägtes subglandulares Gewebe ist bei den Gossypien und bei Tecoma radicans vorhanden, ein Drüsengewebe ohne spezielle Sekretionseinrichtungen bei den Polypodiaceen und bei Acacia cornigera. Die Nektarien der untersuchten Oleaceen besitzen außer einem typischen Drüsengewebe eine besonders modifizierte sezernierende Oberfläche.

Gefäßbündel innervieren oft extraflorale Nektarien und machen nicht selten $\mathrm{zu}$ deren Gunsten Abweichungen vom normalen Verlauf, z. B. 'bei Acacia comigera, Tecoma radicans; Gefäßbündelendigungen fehlen dagegen den Nektarien der untersuchten Polygonaceen und Gossypien.

An der Entwicklung der extrafloralen Nektarien von Acacia cornigera beteiligt sich außer dem Dermatogen zum großen Teil das Periblem.

Die Nektar sezernierende Oberfläche der bearbeiteten Polygonaceen, Gossypien und Oleaceen besteht aus Trichomen; das schüsselförmige Gebilde bei Tecoma radicans ist gleichfalls ein Trichom.

Die Drüsenhaare der untersuchten Nektarien entstehen sämtlich aus je einer Epidermzelle; sie beginnen ihre Entwicklung bei den Polygnnaceen mit zwei Radialteilungen, bei den Oleaceen und Gossypien mit Tangentialteilungen.

Die ausgebildeten Trichome bestehen stets aus drei Abschnitten: Fuß, Stiel und Köpfchen. Der Stielteil ist bei allen dadurch auffallend, 
daß seine Radialwände verdickt und „verkorkt" sind; diese Verkorkung erfolgt erst unmittelbar vor Beginn der Sekretion (cf. p. 34).

Bei Acacia cornigera und in manchen anderen Fällen wird das I)rüsengewebe von einer Zellschicht umscheidet, deren Membranen die Reaktion auf sogenannte "verholzte" Zellwände geben. Diese Zellschicht ist wahrscheinlich physiologisch gleichwertig der Gesamtheit der Stiele aller Nektariumtrichome bei Polygonaceen, Oleaceen, Gossypium u. s. w.

Dic nektarausscheidenden Trichome haben Homologa in einzelstehenden, an entsprechenden Stellen vorkommenden Drüsenhaaren, die meist schon ausgebildet sind, wenn das Nektarium angelegt wird; diese Einzeltrichome werden zum großen Teil beim Entfalten des betreffenden Organes abgestoßen.

Die definitive Lage des zum Nektarium gehörenden Zellkomplexes kommt bisweilen durch ein Nichtteilnehmen desselben am späteren Wachstum des betreffenden Organs zustande; die Form und Anordnung der Nachbarelemente ist gleichfalls oft auf derartige Wachstumsvorgänge zurückzufïhren.

Die extrafloralen Nektarien der untersuchten Polypodiaceen sind ein bisher unbekannter Typus, indem ein- und dieselbe Drüse gleichzeitig auf der Ober- und Unterseite der Lamina Nektar ausscheiden kann.

Die Körper, welche die sogenannten Gerbstoffreaktionen geben, kommen in extrafloralen Nektarien sehr verbreitet vor; sie füllen das Nektariumgewebe bisweilen so dicht an, daß die Vermutung nahe gerückt wird, es häng'e die Bildung des Zuckers irgendwie damit zusammen.

Diese Gerbstoffe entstehen zumteil bereits in dem noch meristematischen Nektarium.

Es lassen sich Gründe für die Ansicht beibringen, daß extraflorale Nektarien oft ursprünglich als Regulatoren für die Wasserbewegung in den von ihnen besetzten Organen der Pflanze gedient haben oder auch jetzt noch dafür dienen; durch indirekte Anpassung an den Besuch von Tieren sind extraflorale Nektarien wohl nicht selten stärker ausgebildet und modifiziert worden, sodaß allmählich eine mehr oder minder weitgehende Symbiose zwischen Pflanze und Tier zustande gekommen ist.

\section{Literatur.}

A ufrecht, S., Beitrag zur Kenntnis extrafloraler Nektarien. Diss. Zürich 1891. Bary, A. de, Vergleichende Anatomie. Leipzig 1877.

Beccari, O., Piante ospitatrici, ossia piante formicarie della Malesia e della Papuasia. Malesia. Vol. II. Genova 1884-86.

Behrens, W. J., Die Nectarien der Blüthen. Flora 1879.

Belt, Th., The naturalist in Nicaragua. London 1874. (Nach Just, Bot. Jahresber.) 
B erthold, G., Untersuchungen zur Physiologie der pflanzlichen Organisation

I, II. Leipzig 1898, 1904.

Bonnier, G., Les nectaires, étude critique, anatomique et physiologique.

Annales des sciences naturelles, botanique Serie 6, tome 8. Paris 1878.

Burck, W., Beiträge zur Kenntnis der myrmekophilen Pflanzen und der Be-

deutung der extranuptialen Nectarien. Annales du jardin botanique de

Buitenzorg. Volume 10. 1891.

Caspary, J., De nectariis. Diss. Elverfeldae 1848.

Correns, C., Zur Anatomie und Entwicklungsgeschichte der extranuptialen

Nectarien von Dioscorea. Sitzb. der k. Akad. der Wiss. zu Wien. Math.-

naturw. Classe Bd. 97. Abt. 1. Wien 1888.

Czapek, F., Biochemie der Pflanzen I, II. Jena 1905.

Delpino, F., Funzione mirmecofila nel regno vegetale. Memorie della acca-

demia delle scienze. Bologna IV 1886-88.

Ewart, M., On the leaf-glands of Ipomoea paniculata. Annals of botany Vol. 9. London 1895 .

Figdor, W., Über die extranuptialen Nektarien von Pteridium aquilinum.

Oest. bot. Ztg. Bd. 41. 1891.

Goebel, K., Pflanzenbiologische Schilderungen I. Marburg 1889.

- Über Studium und Auffassung der Anpassungserscheinungen bei Pflanzen.

Festrede. München 1898.

- Organographie der Pflanzen. 2. Teil. 2. Heft. 1900.

Hab erlandt, G., Anatomisch-physiologische Untersuchungen über das tropische

Laubblatt. Sitzb. d. Wien. Akad. Math.-Naturw. Cl. Abt. 1, Bd. 103,

104. Wien 1894, 1895.

- Physiologische Pflanzenanatomie. 3. Aufl. Leipzig 1904.

Haupt, H., Zur Sekretionsmechanik der extrafloralen Nektarien. Flora 1902.

Kerner von Marilaun, A., Die Schutzmittel der Blüten gegen unberufene

Gäste. Innsbruck 1879 .

- Pflanzenleben I. Leipzig 1888.

Klercker, J. of, Studien über die Gerbstoffvakuolen. Diss. Tubing. 1888.

Kohl, F., Anat. Physiolog. Untersuchg. der Kalksalze etc. Marburg 1889.

Kurr, J., Untersuchungen über die Bedeutung der Nektarien in den Blumen.

Stuttgart 1833.

Lundström, A., Pflanzenbiologische Studien. Nova acta reg. soc. scient.

Upsala. Ser. 3, Bd. 13. (Nach Just, Bot. Jahresb. 1887, p. 441.)

Moeller, H., Anatomische Untersuchungen über das Vorkommen der Gerbsäure. Ber. d. deutsch. bot. Gesellsch. Bd. 6. 1888.

Morini, F., Contributo all' anatomia ed alla fisiologia dei nettarii estranuziali.

Memorie della accademia di Bologna. 4. Serie, Tome 7. 1886.

Pfeffer, W., Pflanzenphysiologie I. 2. Aufl. Leipzig 1897.

Poulsen, V. A., Om nogle Trikomer og Nektarier. Videnskabelige Meddelelser. Kjöbenhavn 1875. (Franz. Resumé gelesen.)

- Nogle extraflorale Nektarier. ibidem 1897 (Referat im Bot. Centralbl. 1898, p. 454 gelesen).

- Das extraflorale Nektarium bei Batatas edulis. Bot. Ztg. 1877. p. 780.

Rathay, E., Über nektarabsondernde Trichome einiger Melampyrum-Arten. Sitzb. Wien. Akad. Bd. 81. Abt. 1. 1880.

- Über extraflorale Nektarien. Verhandlgn. der zool.-bot. Gesellsch. Wien. Bd. 39. 1889. 
Reinke, J., Beiträge zur Anatomie der an Laubblättern, besonders an den Zähnen derselben vorkommenden Sekretionsorgane. Pringsheims Jahrb. f. wiss. Bot. Bd. 10. 1875.

Schimper, A. F. W., Die Wechselbeziehungen zwischen Pflanzen und Ameisen im tropischen Amerika. Jena 1888.

Solereder, H., Systematische Anatomie der Dicotyledonen. Stuttgart 1899.

Sprengel, Ch. K., Das entdeckte Geheimniss der Natur im Bau und in der Befruchtung der Blumen. Berlin 1793.

Stadler, S., Beiträge zur Kenntnis der Nectarien und Biologie der Blüten. Diss. Zürich 1886.

Warburg, Über Ameisenpflanzen. Biol. Centralbl. Bd. 12. 1892.

Wettstein, R. v., Über die Compositen der österreichisch-ungarischen Flora mit zuckerabscheidenden Hüllschuppen. Sitzb. Wien. Akad. Bd.97. 1889.

Wilson, On the cause of the excretion of water on the surface of nectaries. Diss. in Untersuchungen aus dem bot. Inst. zu Tübingen. Bd. I. 1881. Zacharias, E., Über Sekretbehälter mit verkorkten Membranen. Bot. Ztg. 1879.

Zimmermann, A., Über die extranuptialen Nektarien einiger Fagraea-Arten. Ann. du jardin bot. de Buitenzorg. Bd. 18. 1902.

\section{Figuren.}

Fig. 1-7: Muehlenbeckia sagittifolia Meissn.

Taf. I, Fig. 1: Querschnitt eines sezernierenden Nektariums. $t$ Drüsenhaare, die aus 3 Abschnitten bestehen: $b$ Basalteil, $s$ Stielzellen, $k$ Köpfchen; die einzelnen Trichome werden durch Zwischenräume $l$ getrennt. - $g_{1}, g_{2}$, $g_{3}, g_{4}$, Gefäßbündel des Blattkissens (nicht ausgeführt). Vergr. 90.

" Fig. 2: Tangentialer Flächenschnitt durch den Stielteil eines sezernierenden Trichoms; an der Dicke und Anordnung der Membranen ist die Reihenfolge ihrer Entstehung zu erkennen. Vergr. 320.

" Fig. 3: Erstes Entwicklungsstadium auf einem Querschnitt durch das Blattkissen; $t^{1}$ vergrößerte Epidermzelle, aus der ein Trichom wird; $t^{2}$ Epidermzelle, die sich bereits in Quadranten geteilt hat; die ausgezogene Linie gibt die benachbarte Oberfläche an. Vergr. 200.

"Fig. 4: Gleicher Schnitt eines weiter entwickelten Nektariums; die erste tangentiale Querwand wird angelegt. Vergr. 200.

"Fig. 5: Gleicher Schnitt in einer späteren Phase; die Gliederung in 3 Stockwerke ist erreicht; die Entwicklung schreitet von der Mitte gegen den Rand des Nektariums hin fort. Vergr. 200.

n Fig. 6: Gleicher Schnitt in einem noch späteren Stadium; die Radialteilungen in den einzelnen Zellschichten beginnen; die Trichome überragen die angrenzende Epidermis. Vergr. 200.

" Fig. 7: Längsschnitt durch ein fast ausgebildetes Trichom; im Fuß und Stielteil ist die Teilung korrespondierend erfolgt, die Zwischenräume zwischen den einzelnen Trichomen sind kaum zu sehen; die Trichome erscheinen relativ schmaler und höher, als im ausgebildeten Zustand. Vergr. ca. 213. 
Fig. 8: Polygonum baldschuanicum Regel.

Taf. I, Fig. 8: Einige Trichome des Nektariums im Querschnitt: die Stielzellen $s$ haben verdickte und verkorkte Radialwände; die sehr zartwandigen Köpfchenzellen $k$ lassen eine fächerförmige Anordnung erkennen; zwischen den einzelnen Trichomen Zwischenräume $l$. Vergr. 213.

Fig. 9-12: Gossypium brasiliense Macfad.

" Fig. 9: Tangentialer Flächenschnitt durch das Köpfchen eines Trichoms. Der Umriß ist elliptisch, und zwar ist das Trichom in axialer Richtung gestreckt, die Köpfchenzellen sind sehr zartwandig. Vergr. 380.

" Fig. 10: Einige Trichome längs. $b$ Basalzellen, $s$ Stielzelle, $k$ sezernierende Zellen in Schichten angeordnet. Verdickt und verkorkt sind die Radialmembranen der Stielzelle und die an die freie Oberfläche stoßenden Wände $w$ der sockelartig vorgewölbten Basalzellen.' Vergr. ca. 333.

" Fig. 11: Tangentialer Flächenschnitt durch die tiefste Stelle der die einzelnen Trichome trennenden Zwischenräume. Die Basalteile der einzelnen Drüsenhaare sind deutlich gegen einander abgesetzt, stoßen dicht aneinander in Form axial gestreckter Sechsecke, die z. t. kahnförmig zugespitzt sind. Vergr. ca. 200.

" Fig. 12: Querschnitt durch ein jugendliches Nektarium. $t^{1}$ das jüngste Stadium eines Drüsenhaares, $t^{2}$ das am weitesten entwickelte Trichom; zwischen $t^{1}$ und $t^{2}$ die verschiedenen successiven Phasen. Die punktierte Linie gibt die angrenzende Epidermis an, die kaum über das Nektarium hervorragt. Vergr. ca. 86.

Fig. 13: Gossypium microcarpum Tod.

" Fig. 13: Querschnitt durch die Blattmittelrippe, schematisch. em Emergenz, $t$ nektarabsondernde Trichome.

Fig. 14: Tecoma radicans Juss.

" Fig. 14: Querschnitt durch das Nektarium. $h$ zuleitendes Gewebe, das strahlenförmige Anordnung zeigt; $g$ Gefäßbündel; $b$ Basalteil, $s$ Stielzellen mit verdickten und verkorkten Radialwänden, $k$ Köpfchen des Trichoms; $r$ der nach innen etwas übergreifende Rand des Köpfchenteils; bei $k_{1}$ sind die peripheren Zellen keilförmig eingesetzt, bei $k_{2}$ fächerförmig angeordnet. $c$ Cuticula, die am Rande $r$ zahnförmig zwischen die einzelnen Zellen eingreift. Vergr. 72.

Fig. 15-20: Ligustrum Regelianum Hort. Lemoine.

"Fig. 15: Einige Drüsenhaare längs. $b$ Fußzellen; $s$ Stielzelle mit der charakteristischen Radialwandverdickung $r, k$ Köpfchen. Sämtliche Teile des Trichoms sind plasmareich. Vergr. ca. 300.

"Fig. 16: Tangentialer Flächenschnitt durch die Stielzellen in der Höhe des Verdickungsringes $r$. Bei $s$ besteht der Stiel aus 2 Zellen, deren gemeinschaftliche Radialwand in diesem Falle unverdickt und nicht verkorkt ist. Vergr. 466.

Fig. 17: Tangentialer Flächenschnitt durch das Köpfchen eines Trichoms; die Zellen sind plasmareich, nicht so zartwandig, wie z. B. die von Gossypium (cf. Fig. 9). Vergr. 333.

"Fig. 18: Erstes Stadium der Drüsenhaare, Entwicklung von der Mitte gegen die Peripherie fortschreitend. Querschnitt. Vergr. 266. 
Taf. I, Fig. 19: Querschnitt durch ein junges Blatt mit einem jugendlichen Nektarium. $e p$ angrenzende Epidermis der Blattunterseite; $t$ Drüsenhaare; $n$ Nektariumgewebe; $g$ Gefäßbündel. Die Verdickung der Radialwand des Stieles ist noch nicht ausgebildet. Vergr. 283.

"Fig. 20: Längsschnitt durch ein Einzeltrichom auf der Blattunterseite. Der Fuß $b$ des Drüsenhaares steht in ununterbrochenem Zusammenhang mit dem Gefäßbündel $g$; $s$ Stiel mit der charakteristischen Wandverdickung; ch Chlorophyll. Vergr. 293.

Fig. 21: Drynaria Linnaei Bory.

Fig. 21: Unterseite eines sezernierenden Nektariums in der Flächenansicht. Die benachbarten Epidermzellen $e p$ sind radial auf das Nektarium $n$ zu gestreckt; die Epidermzellen des Nektariums haben gerade Wände, sind relativ klein und lassen die ersten Anlagen von Spaltöffnungen erkennen. Vergr. 83.

\section{Fig. 22: Drynaria rigidula J. Sm.}

Taf. II, Fig. 22: Blatt mit einem sezernierenden Nektarium quer. Das Nektariumgewebe $n$ durchsetzt das Blatt gleichmäßig, die Drüsenzellen sind plasmareich und haben keine nennenswerten Intercellularen. Färbung mit Hämatoxylin. Vergr. ca. 70. Mikrophotographie.

Fig. 23: Drynaria Iinnaei Bory.

" Fig. 23: Tangentialer Flächenschnitt durch die Mitte eines sezernierenden Nektariums. An das Drüsengewebe $n$ gehen Gefäßbündelendigungen $g$ heran; gm Gefäßbündel der Blattmittelrippe. Färbung mit Hämatoxylin. Vergr. 70. Mikrophotographie.

Fig. 24-32: Acacia cornigera Willd.

Fig. 24: Nektarium $n$ makroskopisch; die Drüse ist sattelförmig, in axialer Richtung gestreckt. Die Flügel des Blattstiels biegen im Bereich des Nektariums nach außen aus. Etwas verkleinert.

Fig. 25: Querschnitt durch ein sezernierendes Nektarium. $f$ Fuß des Nektariums; $S$ Scheitel der Drüse; $m$ sklerenchymatisch verdickte, abgerundete Zellen in der Mitte des Nektariums; $n$ Drüsenzellen der Richtung der Stoffwanderung entsprechend gestreckt; $s$ verholzte Scheide, ge Gefäßbündelendigungen an den Seiten. Färbung mit Hämatoxylin. Vergr. 70. Mikrophotographie.

" Fig. 26: Blattstiel im Querschnitt; das Nektarium zeigt an den Seiten eine bogenförmige Ausbiegung $b$ nach außen; die sezernierende Furche ist deutlich ausgeprägt. Schematisch. Vergr. ca. 16.

Fig. 27: Querschnitt durch den Blattstiel oberhalb der sattelförmigen Prominenz. Das Nektarium $n$ schiebt sich kegelförmig noch eine Strecke weit unter das Rindengewebe des Blattstieles; die Drüsenzellen sind radial angeordnet. Schematisch. Vergr. ca. 20.

Fig. 28: Teil des Nektariums stärker vergrößert. $n$ Drüsenzellen, die nach der Richtung der Stoffwanderung gestreckt sind; $s$ verholzte Scheide, ge Gefäßbündelendigung; die Vakuolen in $n$ und $s$ sind mit Gerbstoff angefüllt. Vergr. ca. 320.

" Fig. 29: Scheitel des Nektariums. An der sezernierenden Fläche $S$ ist die Epidermis anatomisch nicht scharf ausgeprägt; an beiden Seiten $l$ sind die Zellwände verdickt und kutinisiert. Vergr. 320. 
Taf. II, Fig. 30: Schema für die Verzweigung der Gefäßbündel im Bereiche des Nektariums ; zugrunde liegt ein Nektarium, das nur von der Hauptbündelmasse $(g)$ innerviert wird.

" Fig. 31: Jugendliches Stadium der Emergenz em, aus der sich später das Nektarium herausdifferenziert. Die Epidermis ep teilt sich periklin. Der Gerbstoff $g$ beginnt sich von der Epidermis aus zu bilden. $f$ Fuß des Nektariums. $t$ Trichome, die reich an Gerbstoff sind. $p_{1}$ Prokambium des dem Nektarium benachbarten Gefäßbündels, $p_{2}$ Prokambium des Flügels. Vergr. 266.

" Fig. 32: Späteres Stadium. Die primären Gefäße sind angelegt. Die Emergenz $\mathrm{em}$ ist noch bedeutend kleiner als die beiden Flügel al (cf. Fig. 26). Schematisch. Vergr. ca. 40. 


\section{Lebenslauf.}

Als Sohn des Revierförsters Friedrich Schwendt bin ich, Eduard Schwendt, evangelisch-lutherischer Konfession, am 1. Dezember 1881 zu Seggerde, Kreis Gardelegen, geboren. Von Ostern 1893 ab besuchte ich das Königliche Gymnasium zu Rinteln a. W., das ich Ostern 1902 mit dem Zeugnis der Reife verließ, um zunächst die Königliche Technische Hochschule zu Hannover zu beziehen. Von Michaelis 1902 an widmete ich mich auf der Universität Göttingen dem Studium der Naturwissenschaften und Mathematik.

Ich besuchte die Vorlesungen und Übungen folgender Herren :

in Hannover: Jordan, Kiepert, Rodenberg, Roß, Runge, Seubert;

in Göttingen: A brah a m, Baumann, Ber thold, Blumenthal, Ehlers, Hilbert, Kallius, v. Koenen, Liebisch, Minkowski, Müller, Peter, Rhumbler, Riecke, Simon, Verworn, Voigt, Wagner, Wallach.

Allendiesen meinen Lehrern fühle ich mich zu aufrichtigem Danke verpflichtet. 


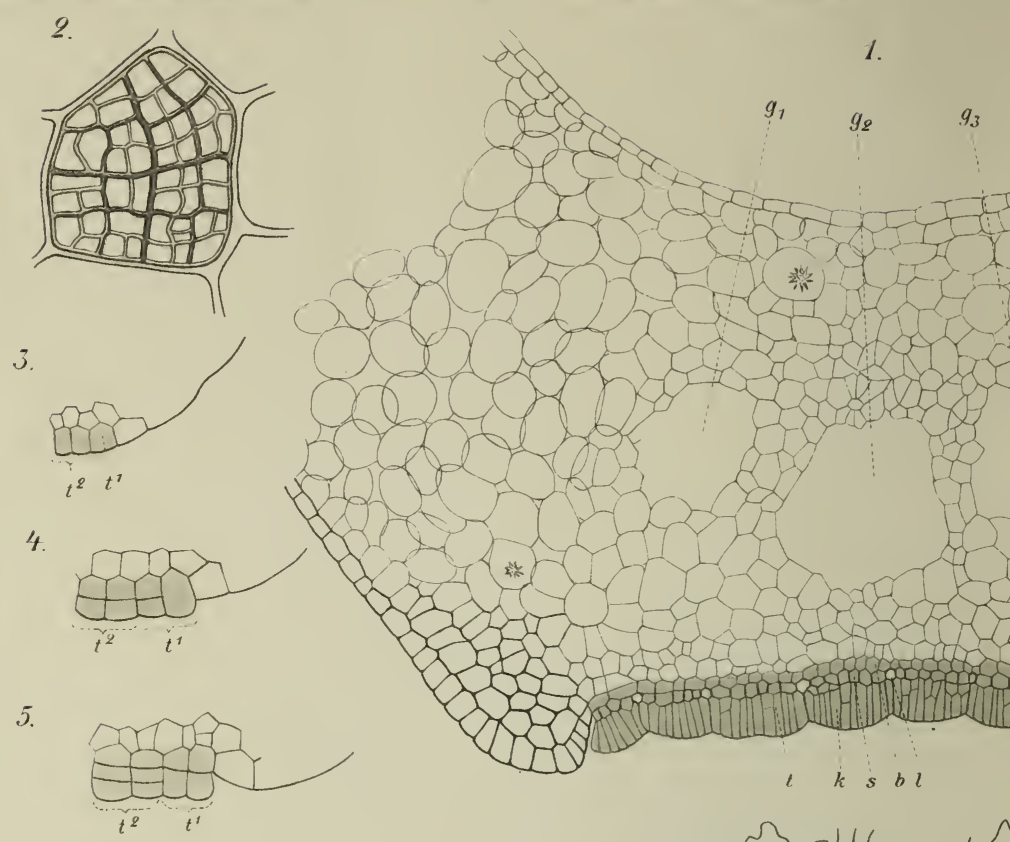

6

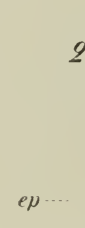

7.
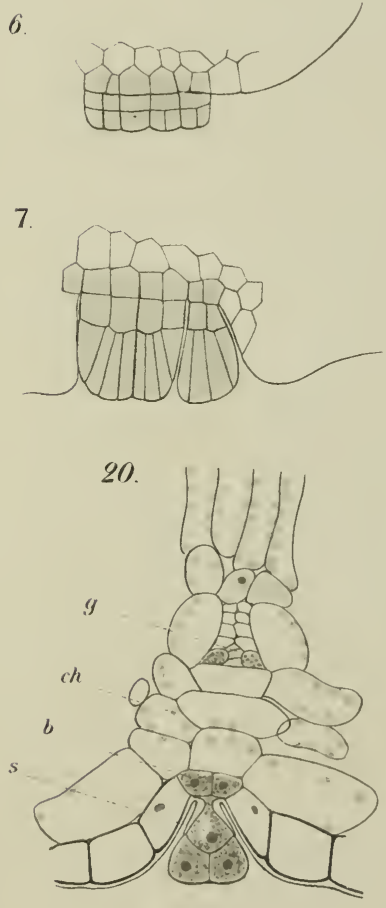

21

$\{\{$ s $\left\{\begin{array}{l}3\} \\ 3\end{array}\right.$

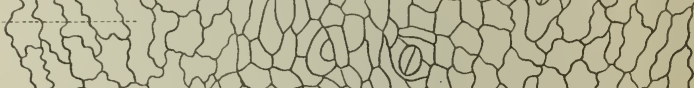
3 s

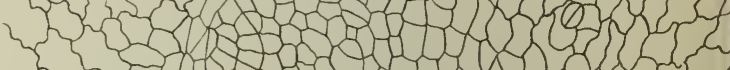
की

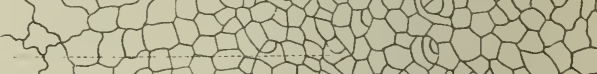

32 rat In th

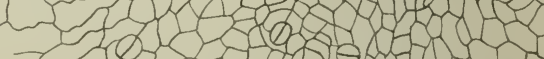
Irtint (n) 
Taf. I.
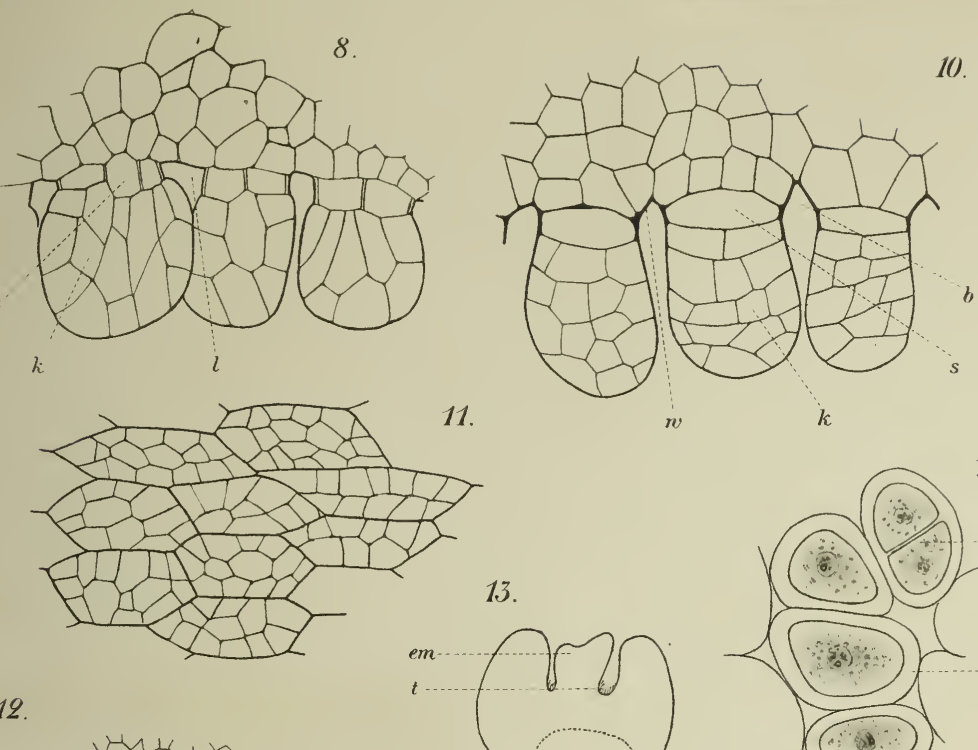

11.

w

k
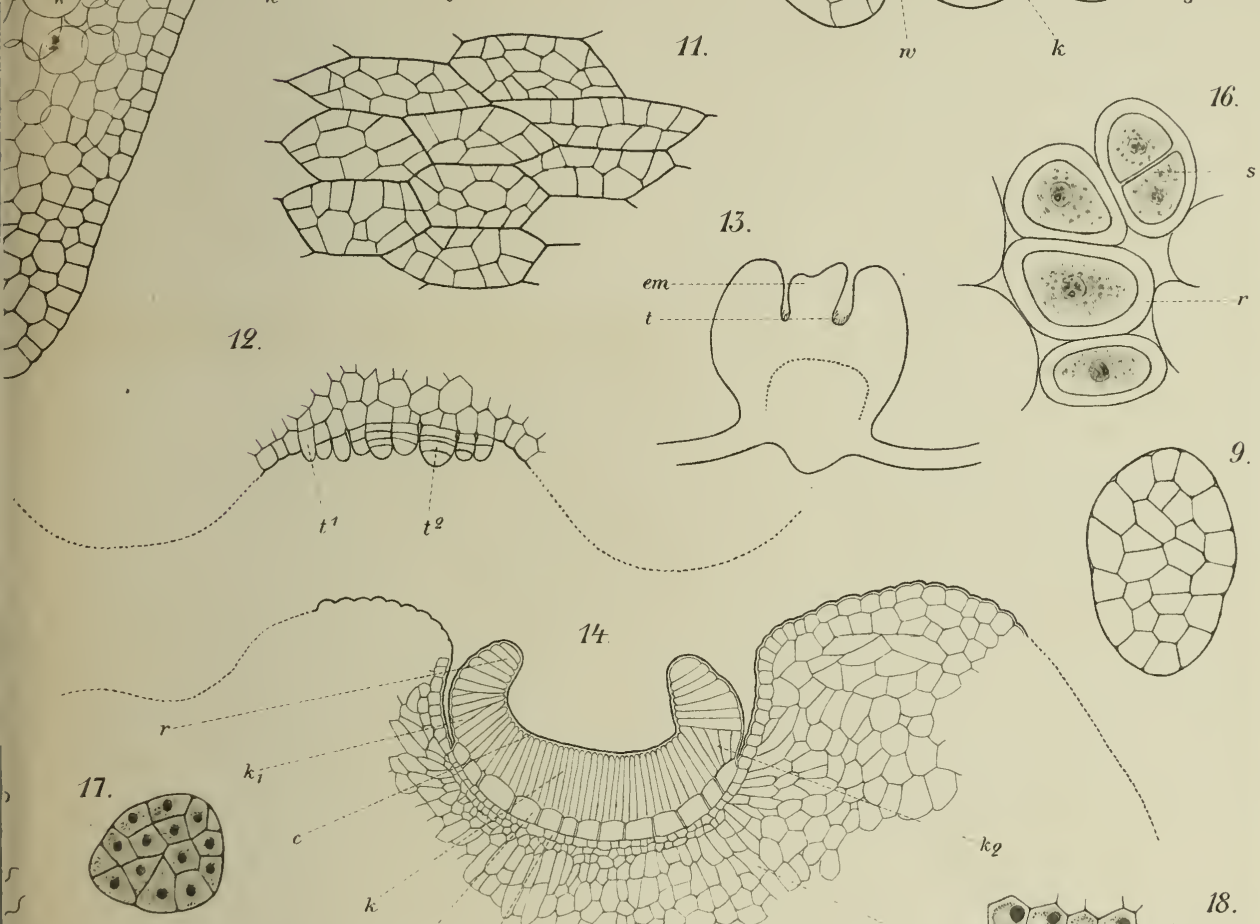

19.
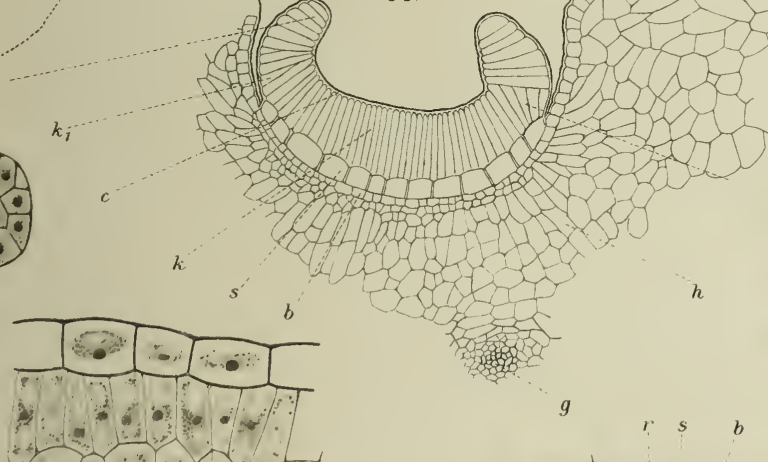



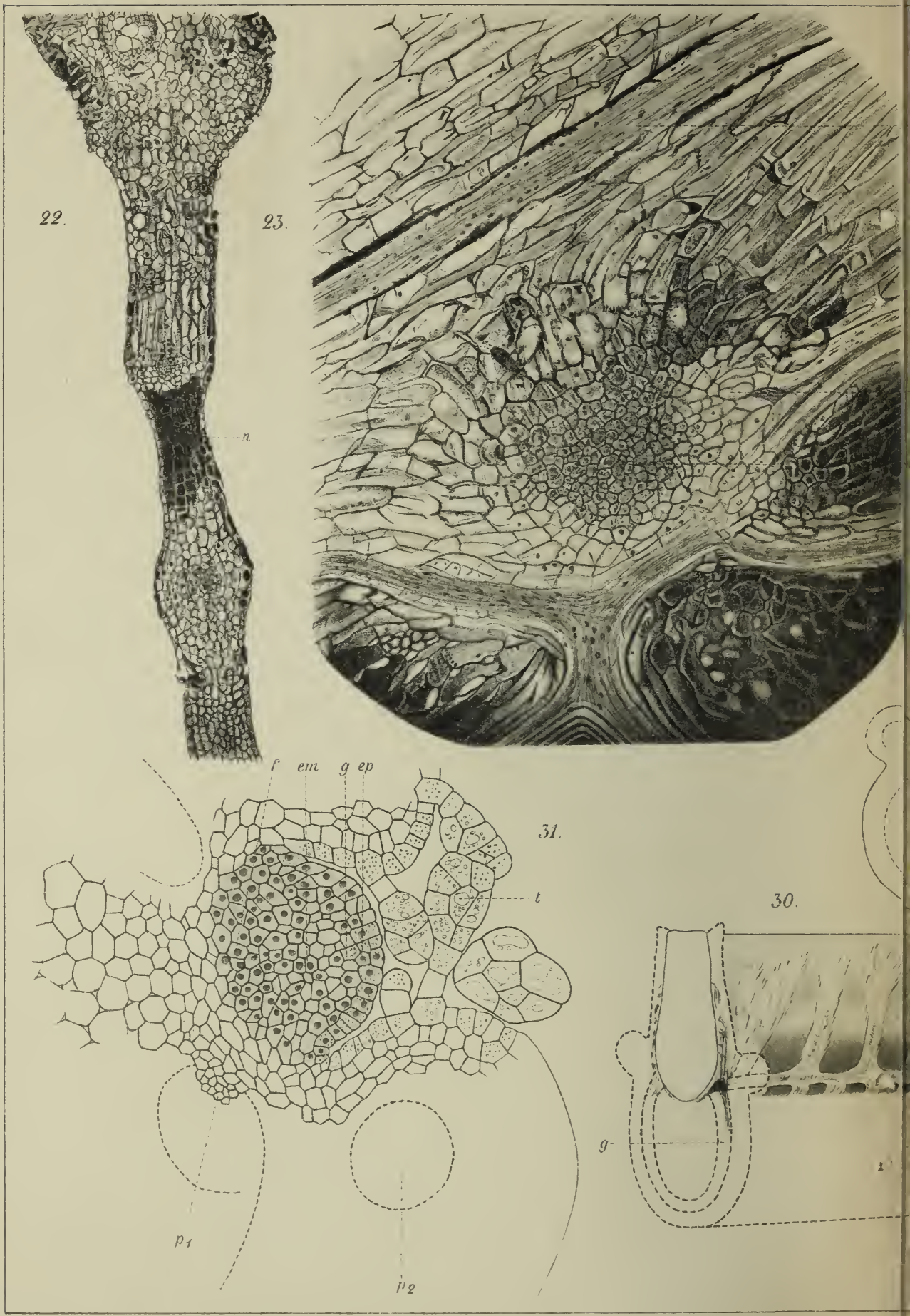


